\title{
Oscillatory Encoding of Visual Stimulus Familiarity
}

\author{
Samuel T. Kissinger, ${ }^{1 \star}$ Alexandr Pak, ${ }^{1 *}$ Yu Tang, ${ }^{1}$ Sotiris C. Masmanidis, ${ }^{2}$ and Alexander A. Chubykin ${ }^{1}$ \\ ${ }^{1}$ Department of Biological Sciences, Purdue Institute for Integrative Neuroscience, Purdue University, West Lafayette, Indiana 47907 and ${ }^{2}$ Department of \\ Neurobiology, David Geffen School of Medicine, California Nanoscience Institute, University of California, Los Angeles, Los Angeles, California 90095
}

Familiarity of the environment changes the way we perceive and encode incoming information. However, the neural substrates underlying this phenomenon are poorly understood. Here we describe a new form of experience-dependent low-frequency oscillations in the primary visual cortex (V1) of awake adult male mice. The oscillations emerged in visually evoked potentials and single-unit activity following repeated visual stimulation. The oscillations were sensitive to the spatial frequency content of a visual stimulus and required the mAChRs for their induction and expression. Finally, ongoing visually evoked $\theta(4-8 \mathrm{~Hz})$ oscillations boost the visually evoked potential amplitude of incoming visual stimuli if the stimuli are presented at the high excitability phase of the oscillations. Our results demonstrate that an oscillatory code can be used to encode familiarity and serves as a gate for oncoming sensory inputs.

Key words: acetylcholine; learning; memory; oscillation; silicon probes; $\theta$

\section{Significance Statement}

Previous experience can influence the processing of incoming sensory information by the brain and alter perception. However, the mechanistic understanding of how this process takes place is lacking. We have discovered that persistent low-frequency oscillations in the primary visual cortex encode information about familiarity and the spatial frequency of the stimulus. These familiarity evoked oscillations influence neuronal responses to the oncoming stimuli in a way that depends on the oscillation phase. Our work demonstrates a new mechanism of visual stimulus feature detection and learning.

\section{Introduction}

Perception and processing of sensory information are critical for animal survival. Efficient strategies are necessary to recognize and differentiate among various visual contexts and stimuli. This recognition and differentiation of information require the segregation of familiar and novel sensory inputs. However, the neural substrates underlying these processes are poorly understood. Visual familiarity arises as animals experience particular visual stimuli, and manifests as changes in neural activity from the naive state within the primary visual cortex (V1). Prior research in this area has primarily focused on the analysis of neuronal responses time-locked to the visual stimuli. Previous visual experience can

Received Dec. 28, 2017; revised June 2, 2018; accepted June 4, 2018

Author contributions:S.T.K., A.P., and A.A.C. designed research; S.T.K., A.P., and Y.T. performed research; S.C.M. contributed unpublished reagents/analytic tools; S.T.K., A.P., Y.T., and A.A.C. analyzed data; S.T.K., A.P., and A.A.C. wrote the paper.

This work was supported by Whitehall Foundation Research Grant, Showalter Trust Young Investigator Grant, and National Institute of Mental Health Grant R01 MH116500 to A.A.C. S.C.M. was supported by a 2014 McKnight Technical Innovations in Neuroscience Award. We thank Mang Gao and Yevgenia Kozorovitskiy for useful discussions and comments.

The authors declare no competing financial interests.

*S.T.K. and A.P. contributed equally to this study as co-first authors.

Correspondence should be addressed to Dr. Alexander A. Chubykin, Department of Biological Sciences, Purdue Institute for Integrative Neuroscience, Purdue University, West Lafayette, IN 47907. E-mail: chubykin@purdue.edu. DOI:10.1523/JNEUROSCI.3646-17.2018

Copyright $\odot 2018$ the authors $\quad 0270-6474 / 18 / 386223-18 \$ 15.00 / 0$ increase the stimulus selectivity of time-locked neural activity in V1 of awake mice (Poort et al., 2015). Similarly, repetitive presentation of phase-reversing gratings to awake mice over several days results in significant potentiation of visually evoked potentials (VEPs) in V1 specifically to the trained stimulus (Frenkel et al., 2006; Cooke et al., 2015). VEP amplitude has also been shown to report learned sequences of visual stimuli, where presentation of a familiar sequence elicits larger amplitude time-locked VEPs compared with a novel sequence (Gavornik and Bear, 2014).

While less well characterized, persistent oscillatory activity lasting beyond visual stimulation may also arise in an experiencedependent manner and potentially reflect stimulus familiarity. Several functions have been attributed to oscillatory activity of neurons in the brain, including information processing, information propagation, and synchronization between brain areas (Salazar et al., 2012; Karalis et al., 2016; Chan et al., 2017; Durkin et al., 2017). Oscillatory activity within particular frequency bands correlates with cognitive load, attention, and other higher-order brain functions (Jensen and Tesche, 2002; Womelsdorf et al., 2006; Voloh et al., 2015; Friese et al., 2016; Mussel et al., 2016). Lowfrequency oscillations are pronounced during sleeping states (Besedovsky et al., 2017; Durkin et al., 2017; Kim et al., 2017). However, recent evidence has also demonstrated the prominence of lowfrequency oscillations in awake states during the perception of visual stimulation (Lee et al., 2005; Einstein et al., 2017). More- 
over, persistent spiking in the $\theta$ oscillation range $(4-8 \mathrm{~Hz})$ emerges in $\mathrm{V} 1$ after the presentation of a visual cue to report the time of a reward delivery (Shuler and Bear, 2006; Zold and Hussain Shuler, 2015) and during the delayed part of visual cuereward working memory tasks (Lee et al., 2005). Thus far, persistent oscillatory activity in V1 has been hypothesized to encode information other than the characteristic features of a visual stimulus, potentially reflecting experience-dependent changes in neural population activity related to the task. It is also plausible that the persistent oscillatory activity induced by previous visual stimulus may interact with new oncoming stimuli. Currently, we do not understand how this oscillatory activity is generated or encoded, nor do we understand how it affects the visual processing of oncoming stimuli.

Importantly, the possibility that oscillations can encode visual stimulus features has not been formally excluded. For example, it remains unclear whether novel versus familiar visual stimuli can differentially evoke oscillations in V1. Whether stimulus-associated oscillations occur in the absence of reward-induced plasticity also remains to be determined. Finally, the necessary and sufficient visual stimulus features that can drive oscillatory activity are unknown. Here, we find that perceptual training leads to the emergence and potentiation of persistent low-frequency oscillations in both local field potentials (LFPs) and single-unit recordings. Consistent with other familiarity-based rhythms in V1 (Chubykin et al., 2013; Zold and Hussain Shuler, 2015), the acquisition and expression of these oscillations depend on the cholinergic muscarinic receptors. The spatial frequency (SF) content of a visual stimulus appears to be the critical factor governing the generation of oscillatory activity. Finally, we demonstrate that familiar stimuli can influence responses to oncoming stimuli, based on the excitability phase at which the subsequent stimuli are presented.

\section{Materials and Methods}

Key resources. Key resources are listed in Table 1.

Mice. All animal use was approved by the Purdue University animal care and use committee. Mice were housed on a $12 \mathrm{~h}$ light/dark cycle, with full access to food and water. Male C57BL/6 mice (The Jackson Laboratory; age postnatal day 60-65) were used for all experiments.

Surgical procedures. At $\sim \mathrm{P} 55$, littermate-matched C57BL/6 mice were selected for surgery. Anesthesia was induced at $5 \%$ inhaled isoflurane (in oxygen) and maintained at 1.5\% during surgery. The skin above the skull was shaved and sterilized with Dynarex ethanol wipes before incision. Ophthalmic ointment was applied to the eyes, and animals were head fixed (with ear bars) in a motorized stereotaxic apparatus (Neurostar) before the surgery. A small incision was made along the midline, then expanded laterally to expose the lambda and bregma skull sutures; $3 \%$ $\mathrm{H}_{2} \mathrm{O}_{2}$ was applied gently with a cotton swab to sterilize and expose the remaining connective tissue on the skull. The underlying connective tissue was removed, and the skull was dried with sterile cotton swabs. Neurostar stereodrive software was used to mark coordinates to target the binocular visual cortex (from lambda: anteroposterior $0.8 \mathrm{~mm}$, mediolateral $\pm 3.2 \mathrm{~mm}$ ). Shallow lines were scratched into the surface of the skull in a checkboard pattern to increase the surface area and facilitate strong bonding of metabond bone cement. A headpost $9.5 \mathrm{~mm}$ in length was glued with cyanoacrylate $3.5 \mathrm{~mm}$ anterior to bregma along the midline. A $1.5 \mathrm{~mm}$ tungsten reference wire soldered to a 0.79 - $\mathrm{mm}$-diameter gold-plated pin was inserted $0.2 \mathrm{~mm}$ forward of the bregma skull suture as a reference and glued in place with cyanoacrylate. Zip-kicker accelerant was used to reduce the drying time of the cyanoacrylate. A small drop of Kwik-Cast Silicone Elastomer was placed above the target coordinates marked on the skull to seal the area until the recording day. Metabond was then used to seal the remaining exposed skull and form a head cap. After a day of recovery, awake mice began habituation to the head- fixation apparatus for a minimum of $4 \mathrm{~d}(90 \mathrm{~min} / \mathrm{d})$. The apparatus consists of an immobile tube that secures the mouse on a raised platform $16.51 \mathrm{~cm}$ directly in front of and centered on a $47.63 \mathrm{~cm} \times 26.99 \mathrm{~cm}$ monitor screen and a bar to hold the surgically implanted headpost. On the first day of habituation, some mice attempted to run to escape head fixation. By the third or fourth day of habituation and during recording sessions, we did not observe any attempts to run. Mice also exhibited grooming behavior by the third or fourth day of habituation. On the fifth day $(\sim$ P60), a craniotomy was made above the visual cortex of a single hemisphere during $5 \mathrm{~min}$ of inhaled anesthesia ( $1.5 \%$ isoflurane) in the stereotaxic apparatus. Mice were then head fixed, and a 64 channel silicon electrode was inserted normal to the surface of the binocular area of the primary visual cortex (anteroposterior $0.8 \mathrm{~mm}$, mediolateral \pm 3.2 $\mathrm{mm}$, dorsoventral $1.0 \mathrm{~mm}$ from lambda). Before acquiring data, additional time $(30 \mathrm{~min})$ after insertion was allowed for the probe to settle and for animals to fully awaken from anesthesia. ACSF was added dropwise above the craniotomy every $20 \mathrm{~min}$ to prevent cortical drying. After a recording session, the recording hemisphere was resealed with KwikCast Silicone Elastomer.

Perfusions and histology. Mice received intraperitoneal injections of a $100 \mathrm{mg} / \mathrm{kg}$ ketamine and $16 \mathrm{mg} / \mathrm{kg}$ xylazine solution for anesthesia. A shallow incision was made below the rib cage to expose the inner cavity of the peritoneum. The lateral sides of the rib cage were cut, and the diaphragm was removed. The rib cage was peeled back, and any remaining connective tissue was removed to expose the heart. A 25 gauge needle was inserted into the left ventricle, and a small incision was made into the right atrium. Blood was forced out of the animal via gravity fed $1 \times$ PBS through the needle. The animal was then perfused with $4 \%$ paraformaldehyde (PFA) to fix the tissue. The animal was decapitated, and the Metabond cap was removed by carefully cutting the cap border and peeling it away from the skull. Shallow cuts were made along the midline, the lambda suture, and the bregma suture to peel back the skull and remove the brain. After extraction, the brain was allowed to sit in $4 \%$ PFA for $24 \mathrm{~h}$ before making $100-\mu \mathrm{m}$-thick coronal sections. The electrode track was then visualized by light microscopy to verify the electrode placement in V1, according to landmarks shown in a mouse brain reference atlas (Neurostar stereotaxic mouse brain atlas, Allen mouse brain atlas).

Visual stimulation. Open source Python-based psychology software (PsychoPy) was used to present visual stimuli. Control gray screen was created using the color space "gray." The mean luminance of the monitor was $73 \mathrm{~cd} / \mathrm{m}^{2}$. After a day of recovery, mice began habituation to the head-fixation apparatus for a minimum of $4 \mathrm{~d}$. During habituation, mice viewed a control gray screen for $90 \mathrm{~min}$ per day. Our standard recording strategy (see Fig. 1) was to show mice single $0.2 \mathrm{~s}$ sinusoidal drifting gratings $(\mathrm{SF}=0.03$ cycles per degree of visual angle, temporal frequency $=3 \mathrm{~Hz}$, speed $=100 \mathrm{deg} / \mathrm{s}$, oriented and drifting at an angle of 150 degrees) for 20 trials in pretraining recordings. Gray screen was presented for $0.5 \mathrm{~s}$ before stimulus onset to serve as a baseline with a total recording time of $2.5 \mathrm{~s}$ or $4.0 \mathrm{~s}$ for each trial, with an intertrial interval of $8 \mathrm{~s}$. Mice were then trained to a pair of stimuli, where the first stimulus (same as described above) is followed by a second stimulus with the same spatial and temporal frequency, but oriented and drifting at an angle of 210 degrees. Animals were trained to this pair of stimuli 200 times in 30 min each day for $4 \mathrm{~d}$. Post-training recordings included the same visual stimulation paradigm as pretraining. In some mice, we also made recordings of the training stimulus after training ( 12 mice from Fig. 1 trained to $1.0 \mathrm{~s}$ interval; 6 mice from Fig. 1 trained to $2.0 \mathrm{~s}$ interval). For SF experiments (see Figs. 9, 10), five visual stimuli were generated by bandpass filtering (PsychoPy filters) random noise with highest power in 0.01 , $0.02,0.04,0.08$, and $0.14 \mathrm{cpd}$. The following bandpass cutin and cutoff values were used to generate stimuli: SF $0.01=(0.00083,0.00086)$, SF $0.02=(0.00167,0.00170)$, SF $0.04=(0.00333,0.00336)$, SF $0.08=$ $(0.00667,0.00670)$, and SF $0.14=(0.01167,0.01170)$.

Data acquisition and Python packages. Recordings were made using 64 channel silicon probes, $1.05 \mathrm{~mm}$ in length, with channels separated 25-50 $\mu \mathrm{m}$ vertically and 16-20 $\mu \mathrm{m}$ horizontally (Shobe et al., 2015). Recordings were made in sets of 20 trials, 2.5 or $4.0 \mathrm{~s}$ in duration. Raw traces and bandpass-filtered units $(300-6000 \mathrm{~Hz})$ were digitized at 30 
Table 1. Reagents and sources

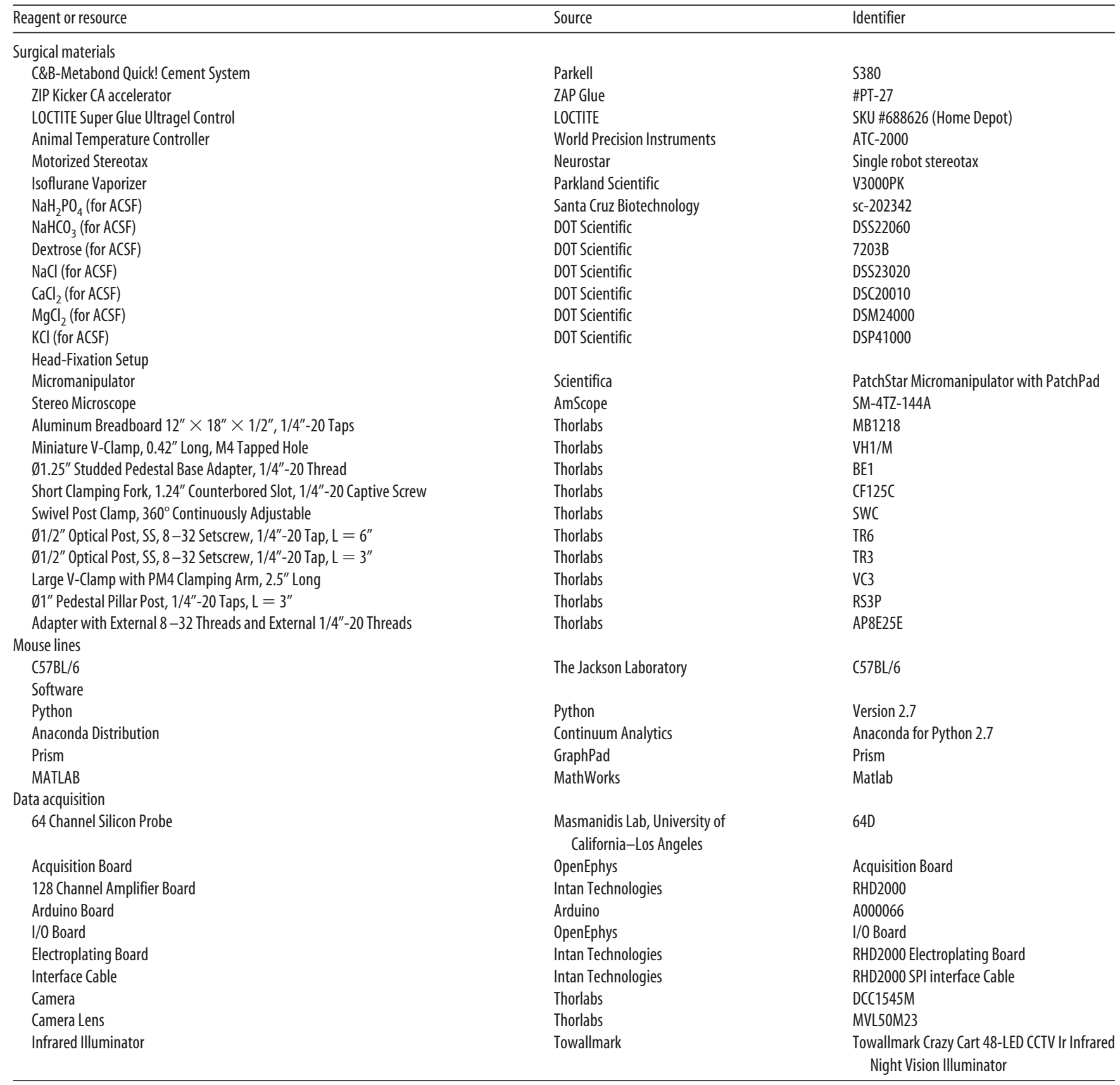

$\mathrm{kHz}$ and acquired with OpenEphys acquisition hardware and software. LFPs were filtered $(1-300 \mathrm{~Hz})$ from raw traces post hoc. All data were plotted and analyzed with jupyter notebook using custom Python code written in our laboratory. Open source data analysis libraries, including Pandas, Scipy, Matplotlib, Seaborn, and sklearn, were used to analyze and plot the data. Pupillometry recordings were acquired with a Thorlabs DCC1545M camera positioned $\sim 28.5 \mathrm{~cm}$ away from the mouse eye, while the pupil was illuminated with infrared light. The videos were analyzed post hoc.

LFP analysis. Raw traces were down-sampled to $1 \mathrm{kHz}$ and manually inspected for artifacts before further analysis. A notch filter was applied to remove $60 \mathrm{~Hz}$ noise. LFPs were compared between animals by taking the first and strongest trial averaged (20 trials) VEP elicited after visual stimulation (putative layer $4 \mathrm{VEPs}$ ) from each of the 3 channel columns of the silicon probe. Because the probes were inserted normal to the surface of the cortex, we could ascertain the current source density (CSD) profile of visually evoked responses. CSD analysis was performed on the trial averaged VEPs across the cortical depth using the spline iCSD method in python (Mitzdorf, 1985; Aizenman et al., 1996; Pettersen et al., 2006; Leski et al., 2007). To determine the average amplitudes of these trial averaged VEPs between mice, 6 windows of time were used to capture local minima (VEP1, 0.53-0.63 s; VEP2, 0.73-0.83 s; VEP3, 0.93-1.03 s; VEP4, 1.15-1.25 s; VEP5, 1.38-1.48 s; VEP6, 1.65-1.75 s) corresponding to the VEP timings observed in our recordings. Timefrequency analysis was performed by using complex wavelet convolution on trial averaged layer 4 VEPs across mice (Cohen, 2014). We used series of complex wavelets to extract power and phase at each sample point. We used 40 frequencies across a logarithmic range from 2 to $80 \mathrm{~Hz}$, with the number of cycles of the wavelet ranging from 3 to 10 for an optimal time-frequency precision tradeoff. Power was $\mathrm{dB}$ normalized to the baseline period. The mean power was then calculated across $\theta(4-8 \mathrm{~Hz}), \alpha$ $(8-12 \mathrm{~Hz}), \beta(12-30 \mathrm{~Hz})$, and $\gamma(30-70 \mathrm{~Hz})$ frequency ranges.

Spike detection and sorting. We used KiloSort, a template-based clustering algorithm implemented in MATLAB, to detect and sort spikes 
from raw binary data (Pachitariu et al., 2016). We used the default KiloSort parameters but set a threshold of 6 SD for spike detection and initialized the templates from data. Clusters were further manually inspected using the Phy template GUI, and several criteria were used to determine the quality of units to be used for further analysis (Rossant et al., 2016). (1) Units with similarity scores of 1.0 were merged, so long as the shape of the average waveforms and their positions on the channel map matched. (2) In rare cases, units with clearly separated clusters in feature view were split. (3) Any unit with waveforms on all channels was removed. (4) Any unit with $<100$ spikes over an 80 s period (20 trials at $4 \mathrm{~s}$ each) or 60 spikes over a $50 \mathrm{~s}$ period ( 20 trials at $2.5 \mathrm{~s}$ each) was removed as reliable autocorrelograms could not be constructed. (5) Any unit with aberrant waveform shapes was removed. (6) Units without a sufficiently clear gap in the absolute refractory period of auto correlograms were removed. (7) After manual sorting, any units with $>5 \%$ of their interspike intervals violating the absolute refractory period were excluded from analysis. This typically resulted in $\leq 3 \%$ of manually sorting units being eliminated from the analysis. (8) Any units with instantaneous firing rates $>200 \mathrm{~Hz}$ were also removed. Klusta Suite/phy clustering software, a more established clustering algorithm, was also used to validate our results (Rossant et al., 2016). Using Klusta Suite/phy gave the same qualitative results.

Single-unit analysis. Peristimulus time histograms (PSTHs) of singleunit activity were computed using $10 \mathrm{~ms}$ bins and smoothed with a Gaussian kernel (width $=100 \mathrm{~ms}$ ). For heat maps, $z$ scores were calculated by normalizing to the mean firing rate (FR) across all time $(z=$ (FR - mean FR)/SD FR). For population time course line plots, $z$ scores were calculated by normalizing FR to the baseline period $(0-0.5 \mathrm{~s})(z=$ (FR - mean baseline FR)/SD baseline FR). To quantify the duration of oscillations in single units, we applied a peak detection algorithm on the $z$-transformed PSTH with the following criteria: (1) the minimum peak height must be at least 1.5 SD from baseline; (2) the first peak must be within $100 \mathrm{~ms}$ of the stimulus onset; and (3) peaks must be within $200 \mathrm{~ms}$ from one another. Units were grouped into different clusters using K-means, an unsupervised clustering algorithm from the sklearn Python package. The input matrix was the PSTH $z$ score of single units across $0-0.25 \mathrm{~s}$ after stimulus onset. We tried initializing clustering both randomly and with the first principal components, but both methods gave qualitatively the same results. Therefore, we used random initialization on all $k$-means clustered data shown in this report. In the majority of cases, we used simple 2-cluster unbiased $k$-means clustering to separate units into visually excited and visually inhibited units. For Figure 10 scatter plots, only units that significantly responded to at least two different stimuli were included to probe single-cell specificity to familiar versus novel stimuli.

Pupillometry analysis. All recordings of mouse pupil size were analyzed post hoc using custom programs written in Python and using the open source computer vision library OpenCV. We imaged the mouse eye at $400 \times 300$ pixels at $20 \mathrm{~Hz}$ with an infrared (IR) camera and lens (Thorlabs). The eye was illuminated with an $850 \mathrm{~nm}$ IR LED (CMVision IR30). Synchronization with electrophysiology recordings was achieved by Arduino and a custom-written Python script in Psychopy. Acquired videos were analyzed using the OpenCV library in Python. For each video, the ROI that only included eye boundaries was selected. For each frame, we first performed image histogram equalization to improve the contrast of the images followed by a Gaussian blur. To perform image segmentation and separate the pupil from the rest of the eye, we applied binary image thresholding. The morphological transformation function morphologyEx was used to remove noise. This was achieved by first using erosion that removes white noise, followed by dilation to restore the original object boundaries, effectively removing white noise. Then we identified the pupil contours by using the function 'findContours' with a mode (RETR_TREE) and method (CHAIN_APPROX_SIMPLE). We used a minimum enclosing circle to define the pupil and to remove edge artifacts caused by whiskers and the IR illumination. In the rare cases when the artifacts could not be removed, the images were removed from the analysis. Automatic pupil detection was manually verified for each video. Following these procedures, pupil area was extracted. If the eye was not sufficiently illuminated, we could not properly track the pupil and had to exclude those recordings from analysis. All recordings of pupil diameter were baseline normalized and reported as a percentage change from baseline.

Statistical analysis. Statistical analysis was performed in Python and Prism. We used two-sample Kolmogorov-Smirnov tests in Python to compare the distributions of oscillation durations among populations of single units. To account for unequal variance and unequal numbers of units between comparisons, we used a Welch's two-sample $t$ test in Python to compare the mean oscillation durations between two unit populations. A Welch's two-sample $t$ test was also used to compare the mean power, pretraining versus post-training, at each frequency band from time-frequency analysis. A two-way ANOVA was used to compare the main effects of training (pre vs post) and oscillation cycle (1-6) and the interaction effect between training and oscillation cycle on either VEP amplitude or firing rate as shown in Figures 1 and 2. A two-way ANOVA was used to compare the main effects of stimulus orientation $\left(150^{\circ}\right.$ vs $300^{\circ}$ ) and oscillation cycle (1-6) and the interaction effect between stimulus orientation and oscillation cycle on VEP amplitude as shown in Figure 6. A two-way ANOVA was used to study the effect of training and SF along with comparing amplitude and power of oscillations within $\mathrm{SF}=0.04$ and $\mathrm{SF}=0.14$ training sets (see Figs. 9, 10). A paired $t$ test was used in scatter plots to compare single-cell oscillatory activity to familiar versus novel stimuli. A two-way ANOVA was used to compare the main effects of visual stimulus condition (Ctrl, in phase, out of phase) and oscillation cycle and the interaction effect between visual stimulus condition and oscillation cycle on VEP amplitude or firing rate as shown in Figure 11. A Tukey-HSD test was used to adjust for multiple comparisons and obtain $p$ values for each comparison after each ANOVA. We attempted the ANOVA and corresponding Tukey-HSD test in python, R, Prism, and MATLAB. However, each of these programs set a limit for how small of a $p$ value could be reported. Therefore, we reported the exact $p$ values when possible but otherwise reported them as $p<0.001$.

\section{Results}

\section{Visually evoked oscillations in V1 emerge with experience to} visual stimuli

To explore the neural correlates of visual familiarity in V1, we performed head-fixed electrophysiological recordings in awake mice before and after perceptual training (Fig. 1A). Acute extracellular recordings were performed with 64 channel silicon probes spanning the cortical depth (Shobe et al., 2015). Using open source data acquisition software (OpenEphys), we simultaneously recorded LFPs and putative single-unit activity to study visual processing at the level of both population responses and individual neurons (Siegle et al., 2017). We first explored the influences of perceptual training to pairs of stimuli, given the evidence that oscillatory activity in V1 can encode the timing of events predicted by visual cues (Shuler and Bear, 2006; Chubykin et al., 2013; Zold and Hussain Shuler, 2015). A sinusoidal drifting grating $(0.2 \mathrm{~s}$ duration, $\mathrm{SF}=0.03 \mathrm{cpd}$, temporal frequency $=3$ $\mathrm{Hz}$, speed $=100 \mathrm{deg} / \mathrm{s}$ ) was presented as a cue to predict a visually distinct second drifting grating after a short delay ( 1 or $2 \mathrm{~s}$ delay, second stimulus-oriented 60 degrees from the first) (Fig. $1 B$ ). Pretraining recordings in response to the cue were primarily characterized by stimulus-locked responses in layer 4 VEPs, although some low-power oscillatory activity could be seen in some cases (Fig. $1 C$, top, blue trace). After repetitively exposing the animals (perceptual training) to the stimulus pairs for $4 \mathrm{~d}$ (200 presentations/ d), high amplitude oscillations emerged in the layer 4 LFPs in response to the visual cue that persisted beyond the timing of visual stimulation (Fig. $1 C$, top, cyan trace). In extreme cases, up to 6 distinct cycles could be seen that lasted up to $1.0 \mathrm{~s}$ beyond the end of visual stimulation. Averaging the maximum amplitudes of these 6 oscillation cycles across mice revealed that the first cycle corresponding to the time-locked VEP did not significantly change in amplitude after training, similar to the first response 
A

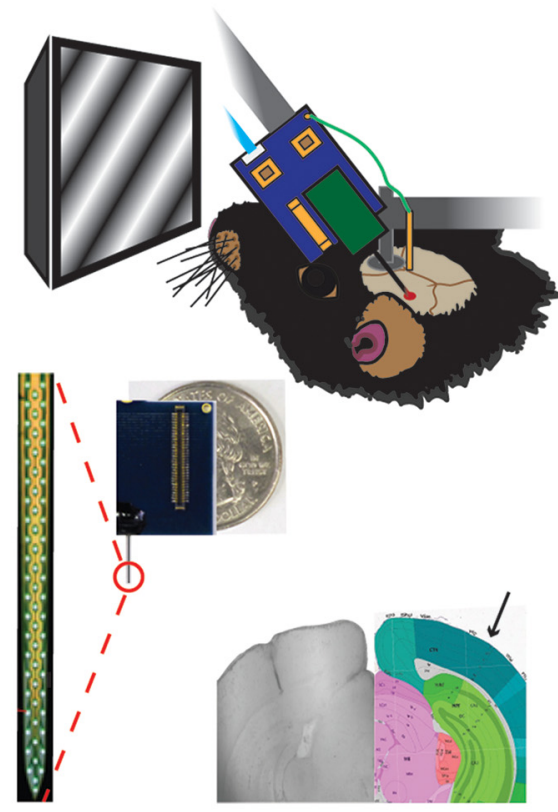

B

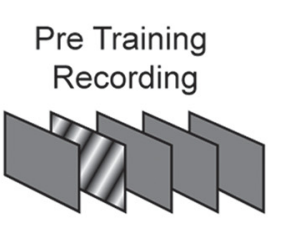

C

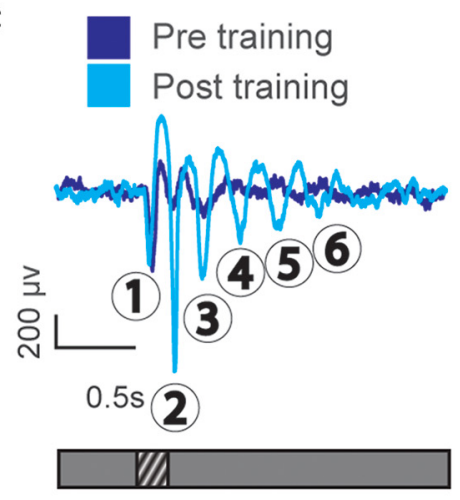

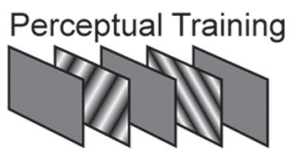

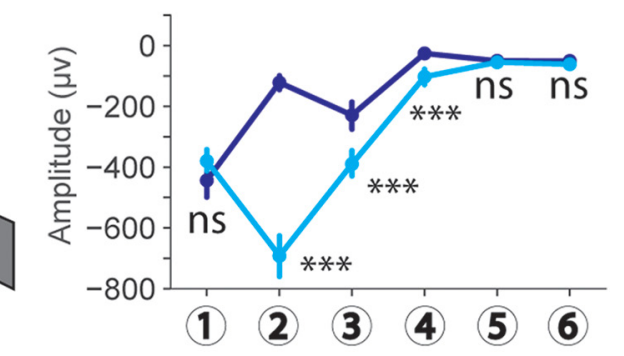

D

E
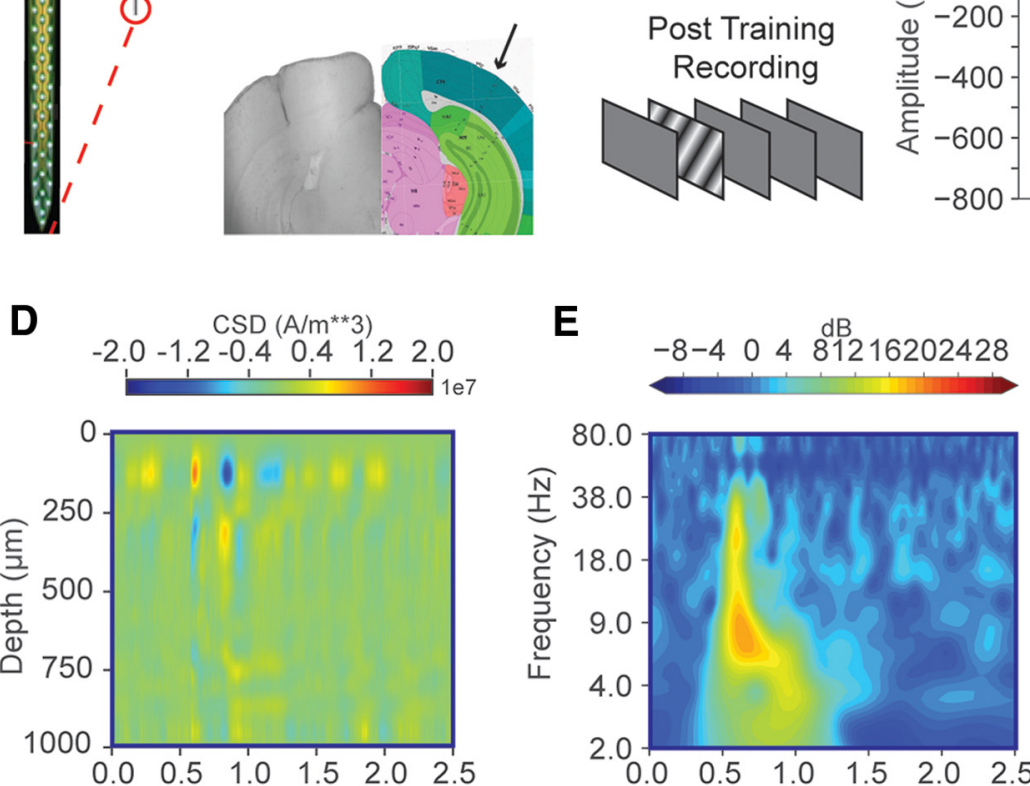

$\mathbf{F}$
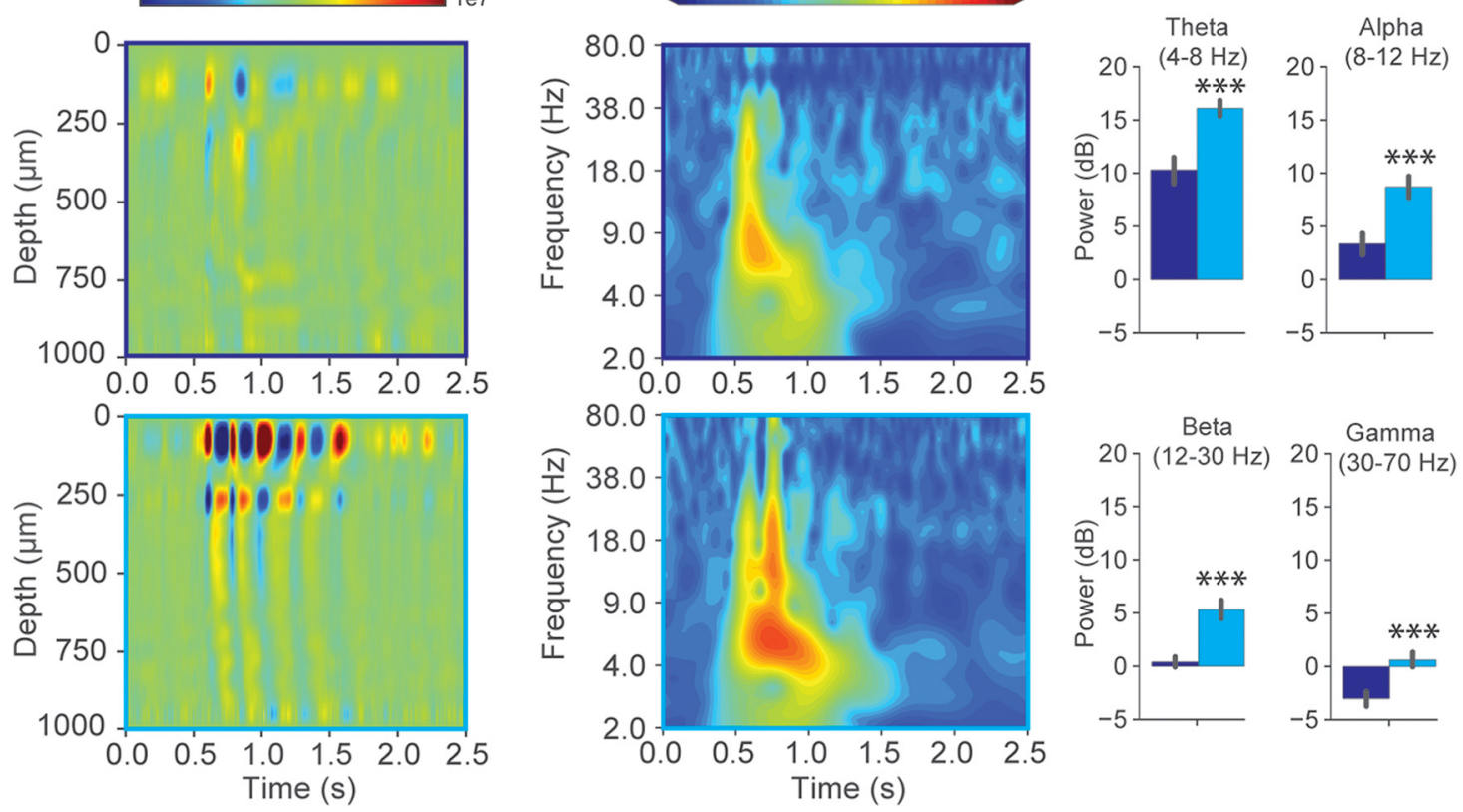

Figure 1. Oscillations emerge with experience to visual stimuli in LFPs. A, Head-fixed electrophysiology in the binocular visual cortex (V1) of awake mice using 64Ch silicon probes. The recording location is verified by light microscopy. $\boldsymbol{B}$, Visual stimulation training paradigm. Mice are recorded in the binocular area of one hemisphere, presented a set of two stimuli separated by a short interval for $4 \mathrm{~d}$ (200 presentations per day), then recorded in the opposite hemisphere after training. C, Top, Representative layer 4 VEPs recorded before (blue) and after (cyan) training. Bottom, Average amplitude across all mice at these VEP timings pretraining and post-training. Two-way ANOVA (Factor 1: pre vs post-training; Factor 2: oscillation cycle) significant interaction between training and oscillation cycle on VEP amplitude $\left(F_{(5,624)}=85.42, p<0.001\right)$. Tukey HSD multiple comparison of means pretraining versus post-training. Selected comparisons: Cycle1: $p=0.30$ (not significant). Cycle2: $p<0.001$. Cycle3: $p<0.001$. Cycle4: $p<0.001$. Cycle5: $p>0.99$ (not significant). Cycle6: $p>0.99$ (not significant) $(N=54$ trial averaged VEPs pretraining, 54 trial averaged VEPs post-training across 18 mice). $\boldsymbol{D}$, Representative CSD analysis, showing the propagation of the oscillation across the full cortical depth (spline iCSD method). $E$, Averaged time-frequency spectrograms pretraining (top) versus post-training (bottom) from all trial-averaged VEPs across 18 mice. $\boldsymbol{F}$, The baseline normalized change in power during the persistent oscillatory period (after stimulus presentation, $0.7-1.2 \mathrm{~s})$ across $\theta(4-8 \mathrm{~Hz}), \alpha(8-12 \mathrm{~Hz})$, $\beta\left(12-30 \mathrm{~Hz}\right.$ ), and $\gamma\left(30-70 \mathrm{~Hz}\right.$ ) frequency bands (paired t test, pretraining (blue) versus post-training (cyan); $\theta: t_{(53)}=6.32, p=5.39 \mathrm{E}-8 ; \alpha: t_{(53)}=8.67, p=9.38 \mathrm{E}-12 ; \beta: t_{(53)}=8.49, p=1.80 \mathrm{E}-11 ; \gamma$. $t_{(53)}=8.28, p=3.86 \mathrm{E}-11 ; N=54$ trial averaged VEPs pretraining, 54 trial averaged VEPs post-training across 18 mice). Error bars indicate SEM. ${ }^{* * *} p<0.001$.

seen during sequential learning paradigms (Gavornik and Bear, 2014) (Fig. 1C, bottom). The second through fourth cycles were significantly potentiated compared with pretraining recordings. After the large increase in the amplitude seen at the second cycle, a decay in amplitude occurred across time until the responses returned to baseline. The oscillations observed in LFP recordings were at the canonical $\theta$ frequency $(5.46 \mathrm{~Hz}, \mathrm{SEM}=0.07 \mathrm{~Hz}$, averaged across 18 mice). There were no significant differences in 

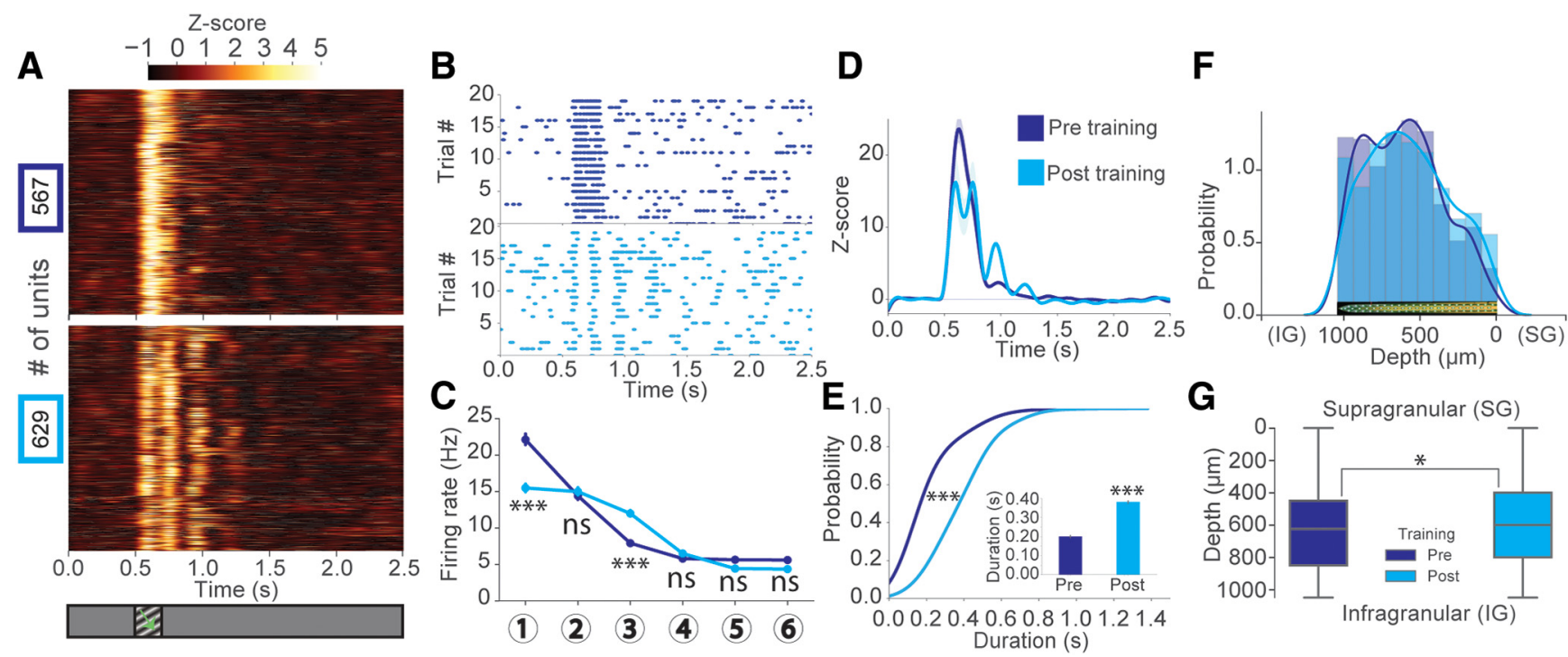

Figure 2. Oscillations emerge with experience to visual stimuli in single-unit activity. $A, z$ score of firing rate for all visually responsive units identified by $k$-means clustering pretraining (top, blue) versus post-training (bottom, cyan) plotted as a heatmap. B, Top, Representative raster plots across 20 trials pretraining (blue) and post-training (cyan) for individual units from the populations shown in $A$. C, Average firing rates at 6 oscillation timings pretraining and post-training for all units shown in $A$. Two-way ANOVA (Factor 1: pre vs post-training; Factor 2: oscillation cycle) significant interaction between training and oscillation cycle on firing rate $\left(F_{(6,8344)}=33.63, p<0.001\right)$. Tukey HSD multiple comparison of means pre versus post. Selected comparisons: Cycle1: $p<0.001$. Cycle2: $p>0.90$ (not significant). Cycle3: $p<0.001$. Cycle4: $p>0.90$ (not significant). Cycle5: $p=0.62$ (not significant). Cycle6: $p=0.59$ (not significant). $N=567$ units pretraining, $N=629$ units post-training across 18 mice. $\boldsymbol{D}$, Population $z$ score (normalized to the baseline period) time course for the units shown in $\boldsymbol{A}$. $\boldsymbol{E}$, Cumulative distribution (CDF) of the duration of $z$ score local maxima 1.5 SD above mean for each unit shown in $\boldsymbol{A}$ (two-sample Kolmogorov-Smirnov test pre vs post, $\mathrm{D}_{(962)}=0.65, p=6.99 \mathrm{E}-92$ ). Inset, Mean peak duration pre versus post (Welch's unequal variances $t$ test, $\left.t_{(962)}=17.79, p=4.39 \mathrm{E}-61\right)(\mathrm{N}=507$ units pretraining after peak detection, $N=457$ units post-training after peak detection across 18 mice). $F$, Distribution of all recorded units pretraining (blue) and post-training (cyan) from the probe across the cortical depth from supragranular (SF) to infragranular (IG) layers. G, Boxplots of the cortical depths of all recorded units pretraining versus post-training. There was a mildly significant difference between the distributions of recorded units across the cortical depth before versus after training (two-sample KolmogorovSmirnov test pre vs post, $\left.\mathrm{D}_{(1899)}=0.06, p=0.03\right)$. Error bars indicate SEM. ${ }^{*} p<0.05,{ }^{* * *} p<0.001$.

the recording depth of the layer 4 VEPs before versus after training, indicating consistent insertions under both conditions (pretraining depth: mean \pm SEM, $380.5 \pm 14.4 \mu \mathrm{m}$; post-training depth: $374.1 \pm 14.6 \mu \mathrm{m}$; 2-sample Kolmogorov-Smirnov test pretraining versus post-training: $p=0.9$, Welch's $t$ test: $p=$ $0.75)$. To quantify the relative changes in the power of persistent (occurring after the stimulus, $0.7-1.2 \mathrm{~s}) \theta(4-8 \mathrm{~Hz})$ oscillations, as well as other common neural oscillation frequency bands, we performed complex wavelet convolution on trial-averaged VEPs across mice (Cohen, 2014). This analysis revealed a significant increase in the power of the $\theta(4-8 \mathrm{~Hz})$ band after training, consistent with the prominent amplitude increases seen in LFPs (Fig. $1 D, E$ ). The power of other frequency bands also increased after training, including $\alpha(8-12 \mathrm{~Hz}), \beta(12-30 \mathrm{~Hz})$, and $\gamma$ $(30-70 \mathrm{~Hz})$ relative to the baseline period. Time-locked responses to visual stimulation displayed clear increases in $\beta$ power compared with baseline in both pretraining and post-training recordings, consistent with other reports of $\beta$ increases in response to grating stimulation (Chen et al., 2015). We observed a dramatic non-stimulus-locked increase in $\beta$ corresponding to the timing of the second cycle that emerged only after training, an interesting result given that the $\beta$ band has been suggested to represent top-down cortical processing (Fig. 1D) (Buschman and Miller, 2007; Schmiedt et al., 2014).

Such prominent oscillations in LFPs suggest a synchronous engagement of a large population of neurons in the local circuit. To examine the firing properties of individual units excited during the oscillation, we compared all visually responsive units before and after training. Individual units displayed stimuluslocked responses before training but displayed sustained periods of spiking activity at $\theta$ frequency only after training (Fig. $2 A, B$ ). Firing rates did not significantly change at the timing of the sec- ond cycle but were significantly increased at the third cycle after training (Fig. 2C). We quantified the duration of oscillatory unit activity based on their mean $z$ score, revealing a significant increase in oscillation duration after training (Fig. 2D,E). These oscillatory units were found across the whole cortical depth. Only mild differences between the cortical depth distributions of all the recorded units could be found pretraining versus post-training, which did not lead to sampling bias (Fig. $2 F, G$ ). Given the intriguing evidence that $\theta$ oscillations may report the time of predicted events in V1, we used our oscillation duration analysis to determine whether training to a pair of salient stimuli influenced oscillation duration. We found that mice trained to a single stimulus also displayed oscillations of comparable duration to those trained to a pair of stimuli (Fig. 3A). Additionally, subsets of animals trained to either $1.0 \mathrm{~s}$ or $2.0 \mathrm{~s}$ interstimulus intervals (ISIs) displayed oscillations after both the first (the "cue") and the second stimulus (Fig. 3B). There was no significant difference in oscillation duration after the first stimulus whether a $1.0 \mathrm{~s}$ or $2.0 \mathrm{~s}$ ISI was presented. Similarly, no significant difference in duration was observed when only the first stimulus (the "cue") was presented between mice trained to these intervals (Fig. 3C). These results indicate that visually evoked $\theta$ oscillations emerge in an experience-dependent manner, but do not explicitly encode interval timing.

\section{The cholinergic system is required for visual experience-dependent oscillations in V1}

V1 receives extensive cholinergic input from the basal forebrain in both rodents and primates (Bigl et al., 1982; Mesulam et al., 1983). Acetylcholine (ACh) is thought to enhance perceptual learning, attention, experience-dependent synaptic plasticity, and has been shown to enhance LTP in L2/3 of binocular visual cortex 
A
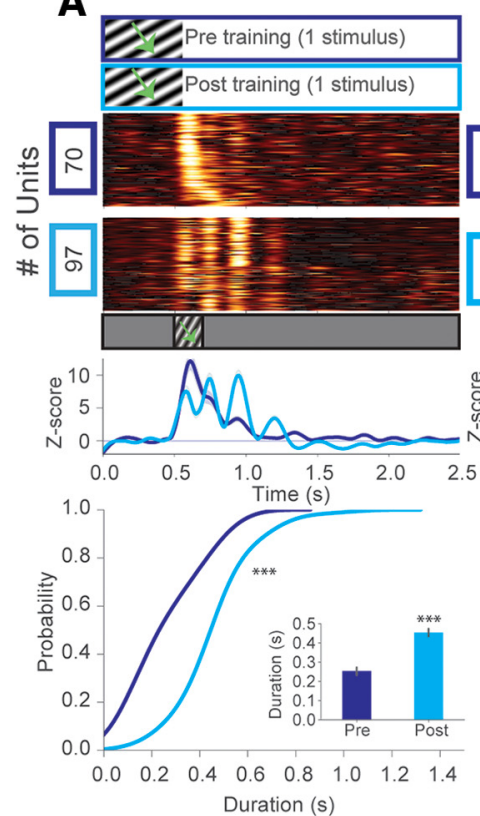

B

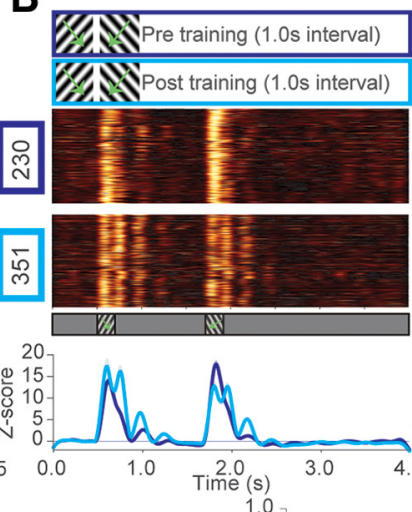

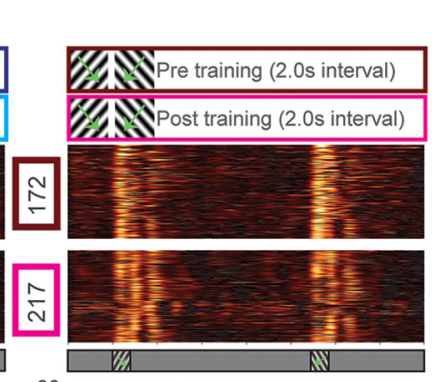

C
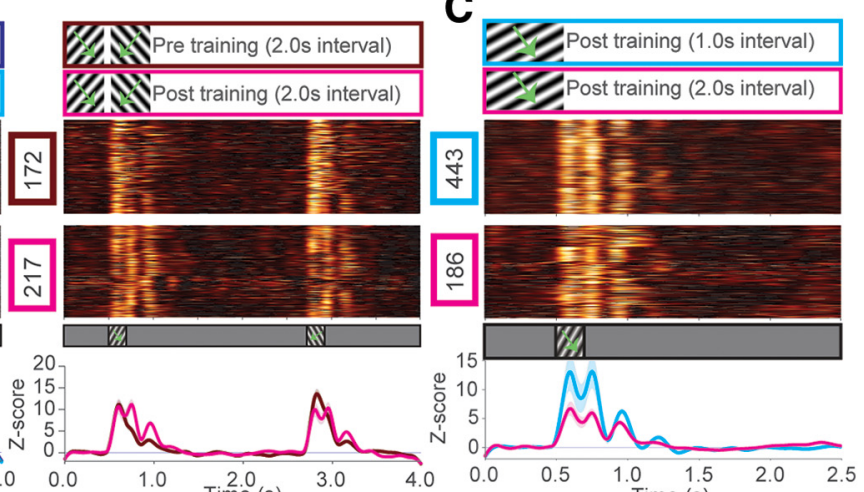
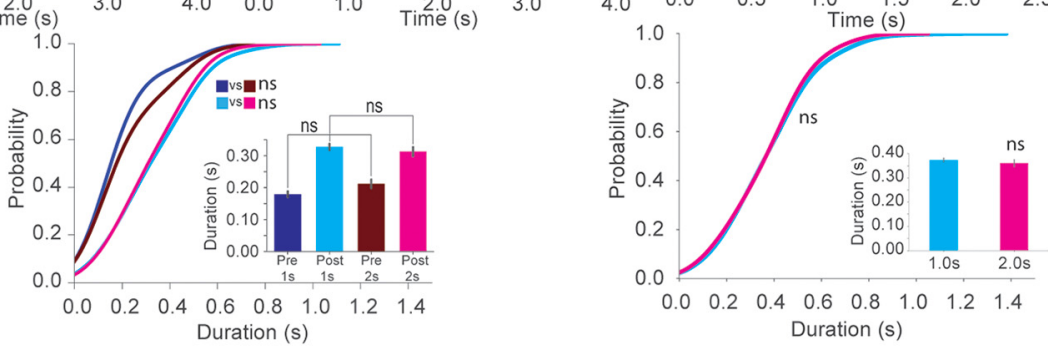

Figure 3. Experience-dependent oscillations in V1 emerge independently of interval training. $A, z$ score of firing rate for all visually excited units from mice trained to a single (instead of paired) sinusoidal drifting visual stimulus ( $\mathrm{SF}=0.03 \mathrm{cpd}$ ) pretraining (blue) and post-training (cyan). Line plots represent a population z score (baseline normalized) for each condition. CDF of oscillation duration: two-sample Kolmogorov-Smirnov test, blue versus cyan: $\mathrm{D}_{(115)}=0.57, p=3.24 \mathrm{E}-9$. Inset, Mean oscillation duration, Welch's unequal variances $t$ test, blue versus cyan: $t_{(115)}=7.0, p=$ $1.8 \mathrm{E}-10$ (units after peak detection): $N=59$ (blue), $N=58$ (cyan), across 2 mice. $B, z$ score of firing rate for all visually excited units from mice trained to a $1.0 \mathrm{~s}$ (blue and cyan; $N=11$ mice) or $2.0 \mathrm{~s}$ (red and magenta; $N=5$ mice) ISI, where both stimuli are presented. For 2 of the 18 mice, the training stimulus was not recorded. Line plots represent a population $z$ score (baseline normalized) for each condition. CDF of oscillation duration: two-sample Kolmogorov-Smirnov test, blue versus red: $D_{(310)}=0.12, p=0.21$; cyan versus magenta: $D_{(387)}=0.13, p=0.08$. Inset, Mean oscillation duration, Welch's unequal variances $t$ test, blue versus red: $t_{(310)}=1.49, p=0.13$; cyan versus magenta: $t_{(387)}=0.81, p=0.41$ (units after peak detection): $N=184$ (blue), $N=277$ (cyan), $N=128$ (red), $N=112$ (magenta). C, z score of firing rate for all visually excited units from mice trained to a $1.0 \mathrm{~s}$ (blue; $N=12$ mice) or $2.0 \mathrm{~s}$ (cyan; $N=6$ mice) ISI, where only the first visual stimulus (the "cue") is presented. No significant difference could be found in the duration of oscillations between mice trained to either interval. CDF of oscillation duration: two-sample Kolmogorov-Smirnov test, blue versus cyan: $\mathrm{D}_{(491)}=0.13, p=0.06$. Inset, Mean oscillation duration, Welch's unequal variances $t$ test, $t_{(491)}=0.71, p=0.47$ (units after peak detection): $N=$ 371 (cyan), $N=122$ (magenta). Error bars indicate SEM. ${ }^{* * *} p<0.001$.

(Gu, 2003; Wilson et al., 2004; McCoy et al., 2008; Kang et al., 2014). To explore the role of mAChRs in the acquisition of visually evoked $\theta$ oscillations, mice received intraperitoneal injections of $3 \mathrm{mg} / \mathrm{kg}$ scopolamine, a nonspecific $\mathrm{mAChR}$ antagonist, 30 min before each training session for $4 \mathrm{~d}$. After $4 \mathrm{~d}$ of drug treatment with training, we did not observe oscillations in these animals. Visual responses were transient and time-locked to the stimulus, similar to those typically seen in pretraining recordings in controls (Fig. 4A). After allowing $24 \mathrm{~h}$ for drug washout followed by 4 additional days of training without scopolamine, oscillatory activity recovered. To test whether $\mathrm{mAChRs}$ are necessary for the expression of $\theta$ oscillations, we trained a separate group of mice for $4 \mathrm{~d}$, followed by a $3 \mathrm{mg} / \mathrm{kg}$ scopolamine injection on the final day $30 \mathrm{~min}$ before the recording session only. Similarly, $\theta$ oscillations were blocked but recovered after 4 additional days of training (Fig. 4B). These results suggest that the cholinergic system, through the activity of muscarinic receptors, is required for both the acquisition and the expression of visually evoked $\theta$ oscillations in V1. This is consistent with other experiencedependent phenomena reported in V1, which have shown that both persistent unit activity and the potentiation of VEPs to familiar visual stimuli are dependent on the cholinergic system (Chubykin et al., 2013; Gavornik and Bear, 2014).

Experience-dependent oscillations report familiarity to visual stimuli in V1

Next, we hypothesized that the motion of the gratings might directly influence the emergent oscillations in V1. To test this notion, mice were trained to $200 \mathrm{~ms}$ static stimuli at the same SF $(0.03 \mathrm{cpd})$ and orientation (150 degrees) as the sinusoidal drifting stimulation seen in Figure 1. Oscillatory activity was significantly increased in duration after training despite the visual stimulus remaining stationary (Fig. 5A). In the same animals, we presented an extended-duration static stimulus ( $800 \mathrm{~ms}$ ) to determine whether the oscillations were sensitive to the duration of the training stimulus (200 ms). This manipulation extended stimulus-evoked persistent firing in units before training but failed to elicit low-frequency oscillations. After training, however, persistent low-frequency oscillations were evoked throughout the entire duration of stimulus presentation and beyond (Fig. 5B). Next, we tested the sensitivity of these oscillations to stimulus novelty. The first parameter we changed was stimulus orientation, considering that other experience-dependent changes in visual responsiveness, such as stimulus-specific response potentiation, are sensitive to orientation (Frenkel et al., 2006; Cooke et al., 2015). Presentation of a novel stimulus orientation (300 degrees vs 150 degrees) to trained mice yielded persistent responses that did not oscillate strongly (Fig. 6A,B). Oscillations in response to the novel stimulus were more evident among VEPs but had decreased magnitude compared with those evoked by the trained stimulus (Fig. 6C,D). However, the oscillations were still present in response to the novel orientation, suggesting that their amplitudes, but not their emergence, are orientation selective.

Next, we tested whether drifting gratings of different spatial frequencies could promote the emergence of these oscillations. Mice trained to drifting gratings of high SF $(0.3 \mathrm{cpd})$ also dis- 

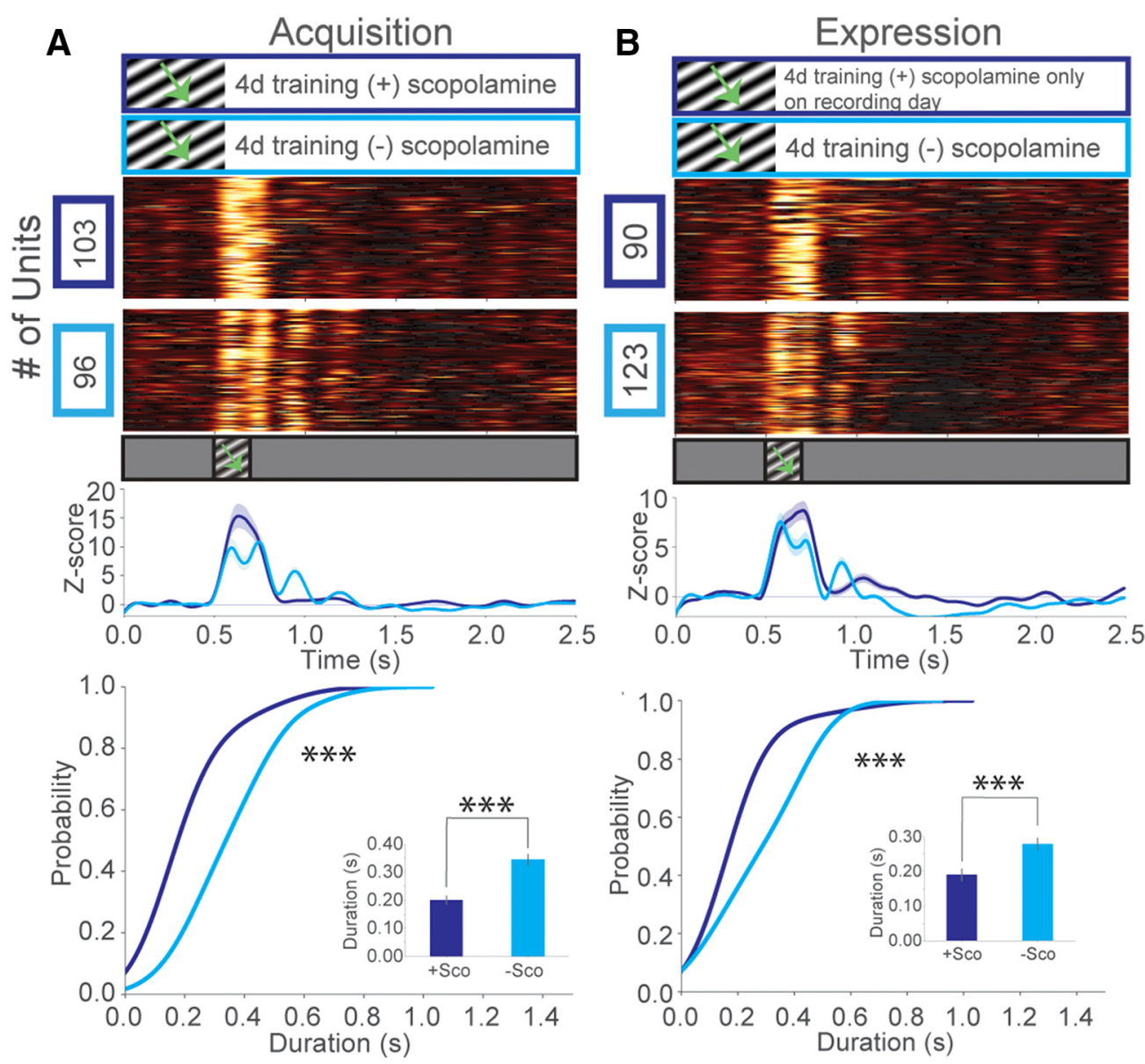

Figure 4. Experience-dependent oscillations in V1 depend on the muscarinic receptors. $A, z$ scores of firing rates for mice trained to an intermediate SF ( $0.03 \mathrm{cpd})$ stimulus for $4 \mathrm{~d}$, with $3 \mathrm{mg} / \mathrm{kg}$ scopolamine delivered $30 \mathrm{~min}$ before each training session (blue). Mice received 4 additional days of training without scopolamine treatment (cyan). Line plots represent a population $z$ score (baseline normalized) for each condition. (DF of oscillation duration: two-sample Kolmogorov-Smirnov test, $D_{(129)}=0.62, p=6.81 E-12$. Inset, Mean oscillation duration, Welch's unequal variances $t$ test, $t_{(129)}=5.77, p=7.99 \mathrm{E}-8$ (units after peak detection: $N=76$ (blue), $N=55$ (cyan), across 4 mice). $B, z$ scores of firing rates for mice trained to an intermediate $S F$ ( 0.03 cpd) stimulus for $4 \mathrm{~d}$, with $3 \mathrm{mg} / \mathrm{kg}$ scopolamine delivered only on the recording day (blue). Mice received 4 additional days of training without scopolamine treatment (cyan). CDF of oscillation duration: two-sample Kolmogorov-Smirnov test, $\mathrm{D}_{(122)}=0.41, p=5.45 \mathrm{E}-5$. Inset, Bar graph of mean oscillation duration Welch's unequal variances $t$ test, $t_{(122)}=3.45, p=7.73 \mathrm{E}-4$ (units after peak detection: $N=45$ (blue), $N=79$ (cyan), across 3 mice). Error bars indicate SEM. ${ }^{* * *} p<0.001$.

played these oscillations (Fig. 7A). Interestingly, when these mice were presented with an intermediate SF $(0.03 \mathrm{cpd})$ drifting grating, we noticed a distinct lack of oscillatory activity. This could be replicated in mice trained to low $S F(0.003 \mathrm{cpd})$ stimuli and presented with a novel stimulus with an intermediate SF $(0.03 \mathrm{cpd})$ (Fig. 7B). To further test whether the presence of the oscillations is specific to a particular type of visual stimuli, we trained mice to a drifting grating $(200 \mathrm{~ms}, \mathrm{SF}=0.03 \mathrm{cpd})$ and displayed a complex grayscale checkerboard stimulus as a novel stimulus (Fig. 7C). Again, oscillations emerged in response to the trained stimulus, but not the novel checkerboard stimulus. Yet, mice could also be trained to the grayscale checkerboard stimulus to elicit oscillations (Fig. 7D). Surprisingly, when we presented an intermediate SF $(0.03 \mathrm{cpd})$ grating as a novel stimulus to these mice, prominent oscillatory activity was elicited, similar to that evoked by the familiar checkerboard stimulus. This is likely because the checkerboard stimulus contains a broad range of spatial features, leading to the cross-activation of the trained circuitry when a simpler grating stimulus was presented. Thus, the spatial complexity of a familiar stimulus may be sufficient to drive oscillatory responses to novel "component" stimuli.

Among VEPs, we consistently noticed that the second cycle of the oscillation was the most potentiated (see Fig. 1D). This ob- servation may reflect the strengthening of an "off" response to the $200 \mathrm{~ms}$ stimulus after training, as it occurs shortly after the stimulus ends. If so, then longer presentations of familiar stimuli should extend the oscillatory period and delay the "off" response accordingly. To explicitly test this prediction, we presented visual stimuli of extended durations (400, 800, and $1600 \mathrm{~ms}$ ) after training to an intermediate SF grating $(200 \mathrm{~ms}, 0.03 \mathrm{cpd})$. In this experiment, a low SF grating $(0.003 \mathrm{cpd})$ was presented as a novel event. Oscillatory activity in both individual units and LFPs persisted across all durations of the stimulus in mice trained to the intermediate SF stimulus (Fig. 8A). The oscillations were abolished when the low SF stimulus was presented as a novel event. We then did the opposite experiment with another group of mice, which were trained to a low SF grating with the intermediate SF grating presented as a novel event (Fig. $8 B$ ). Similarly, the oscillations were present only in response to the familiar low SF grating stimulus. The stimulus-locked oscillations were clearly different in frequency from the persistent oscillations previously observed in response to $200 \mathrm{~ms}$ stimuli at this SF (compare with Fig. $7 B$ ). Given that the oscillations can persist throughout the presentation of familiar long-duration static stimuli (Fig. 5B), we suggest that the mechanism of the familiarity-evoked persistent oscillations is different from that of the visual stimulus-locked 
A
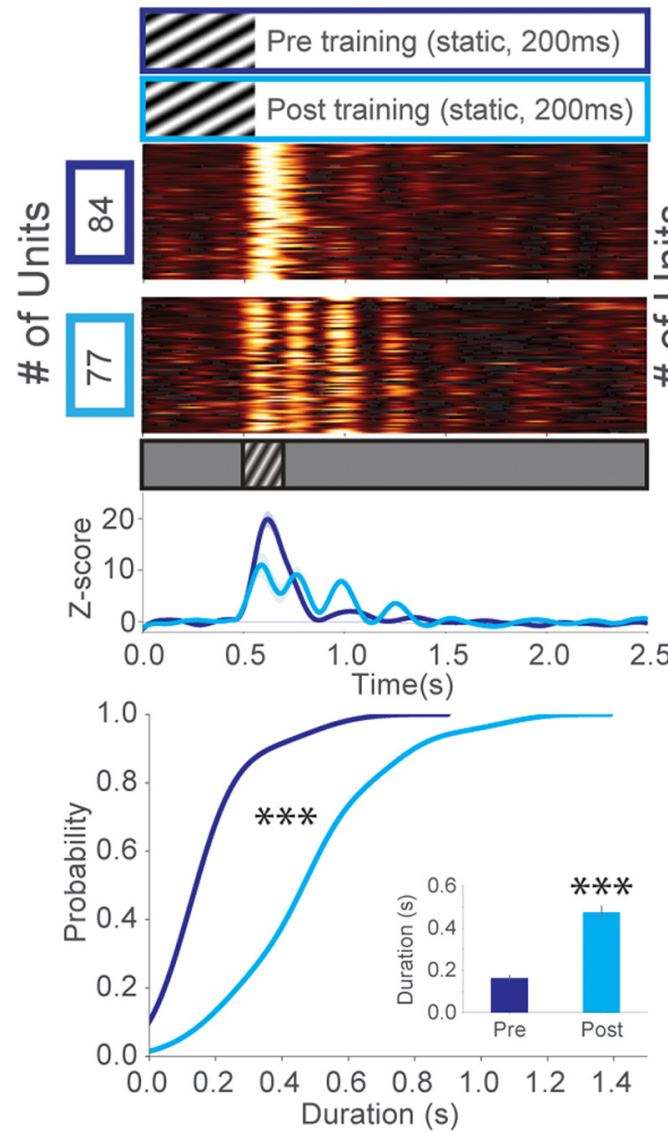

B
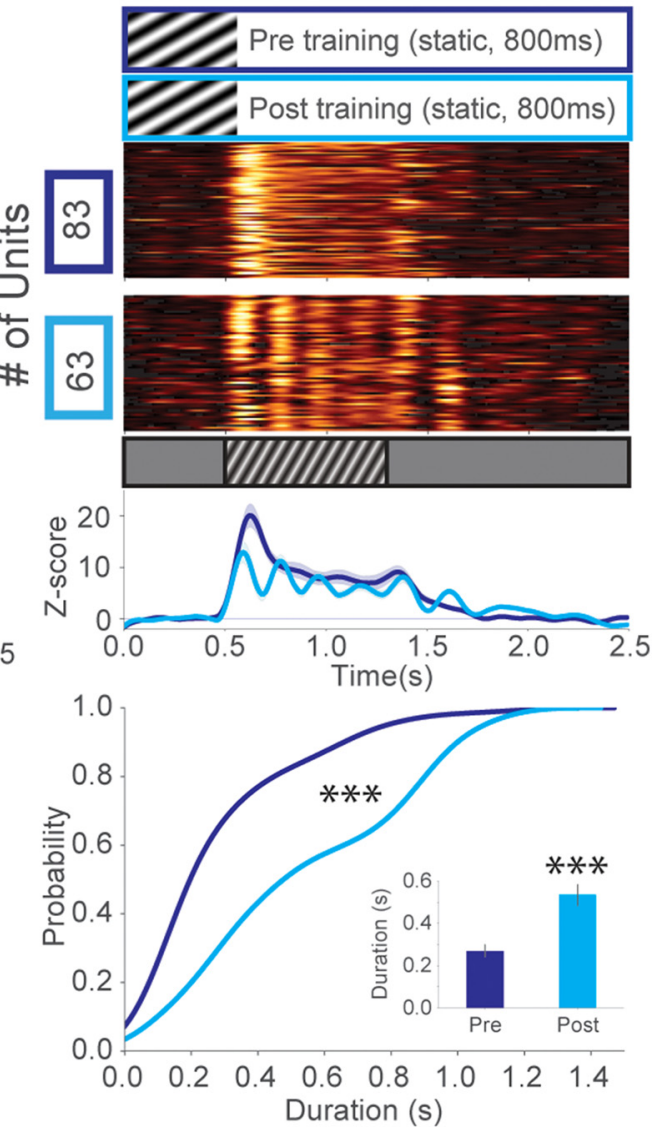

Figure 5. Experience-dependent oscillations in V1 emerge with experience to static visual stimuli. $A, z$ score of firing rate for all visually excited units from mice trained to a static 200 ms visual stimulus ( $\mathrm{SF}=0.03 \mathrm{cpd}$ ) before training (blue) and after training (cyan). Oscillatory activity was observed after training despite the stimulus remaining stationary. Line plots represent a population $z$ score (baseline normalized) for each condition. CDF of oscillation duration: two-sample Kolmogorov-Smirnov test, blue versus cyan: $D_{(136)}=0.80, p=4.36 E-20$. Inset, Mean oscillation duration, Welch's unequal variances $t$ test, $t_{(136)}=9.46, p=1.29 \mathrm{E}-14$ (units after peak detection): $N=80$ (blue), $N=58$ (cyan), across 2 mice. $B$, Extending the duration (to 800 ms) of the static stimulus also extended the duration of the oscillations, despite the fact that the stimulus was not moving or changing in any way during the duration of presentation. Before the animals were trained (to 200 ms stimuli), only persistent firing was observed during stimulus presentation. (DF of oscillation duration: two-sample Kolmogorov-Smirnov test, pretraining (blue) versus post-training (cyan): $\mathrm{D}_{(108)}=0.47, p=6.24 \mathrm{E}-6$. Inset, Mean oscillation duration, Welch's unequal variances $t$ test, $t_{(108)}=4.56, p=1.99 \mathrm{E}-5$ (units after peak detection): $N=65$ (blue), $N=45$ (cyan), across 2 mice. Error bars indicate SEM. ${ }^{* * *} p<0.001$.

oscillations. The second cycle of the oscillation remained highly potentiated regardless of stimulus duration, suggesting that it represents a conserved property of the oscillation that is separate from the delayed "off" response seen at the end of each extended duration. Overall, these results suggest that visually evoked oscillations in V1 emerge with visual experience and may be representative of visual familiarity in a SF-dependent manner.

SF is the major stimulus feature driving the emergence of the oscillations

After the observation that oscillatory activity was not induced by drifting grating stimuli with novel SFs, we decided to further investigate the SF tuning of the oscillations without the confounding factors of orientation or temporal frequency. We generated static stimuli of nonoverlapping SF bands by bandpass filtering sparse noise in a range of frequency bands $(0.01,0.02,0.04,0.08$, or 0.14 cpd) (Fig. 9A). Separate groups of animals were trained to specific SF bands (either $0.2 \mathrm{~s} \mathrm{SF}=0.04 \mathrm{cpd}, n=4$ mice; or $\mathrm{SF}=0.14 \mathrm{cpd}$, $n=6$ mice $)$ the same way as described above ( $4 \mathrm{~d}, 200$ presentations/d), with all five stimuli presented both pretraining and post-training. Pretraining recordings showed that all five stimuli induce stimulus-locked firing of single units and stimulus-locked VEPs, consistent with our pretraining results to grating stimula- tion. After training, we observed the emergence of oscillations in both groups of mice (Fig. 9B). In both cases, however, the amplitude of the oscillations was highest for the trained SF compared with the novel SFs, particularly at the second oscillation cycle (Fig. 9B,C). Time-frequency analysis revealed potentiation of low-frequency oscillations that persisted beyond the stimulus duration in both training groups, especially to the trained SF (Fig. $9 D$, green and purple boxes). Overall, oscillatory activity varied based on how close in SF a stimulus was to the experienced one. We found that low-frequency oscillations were significantly modulated by the trained stimulus compared with novel stimuli within each training group (Fig. 9E).

Next, we analyzed single-unit activity to assess how individual neurons responded to the experienced versus novel stimuli by quantifying the duration of stimulus-induced oscillatory activity. Unit analysis revealed that both single-cell and population level activity could be used to discriminate between the experienced SF band (oscillatory response) and novel stimuli (transient response). On a population level, neural activity reflected our observations from LFP analysis showing that oscillatory activity was specific to the trained SF band (Fig. 10A). We found that the duration of oscillatory activity was significantly longer for the trained stimulus compared with the novel one within each training group (for 
A

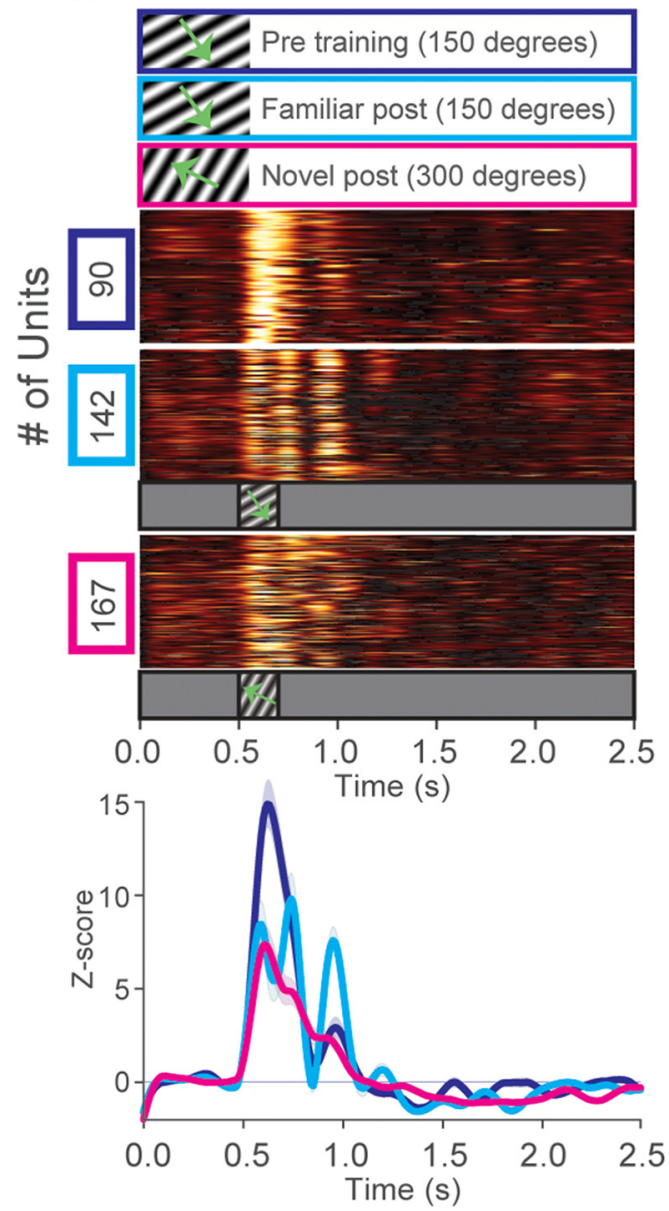

B
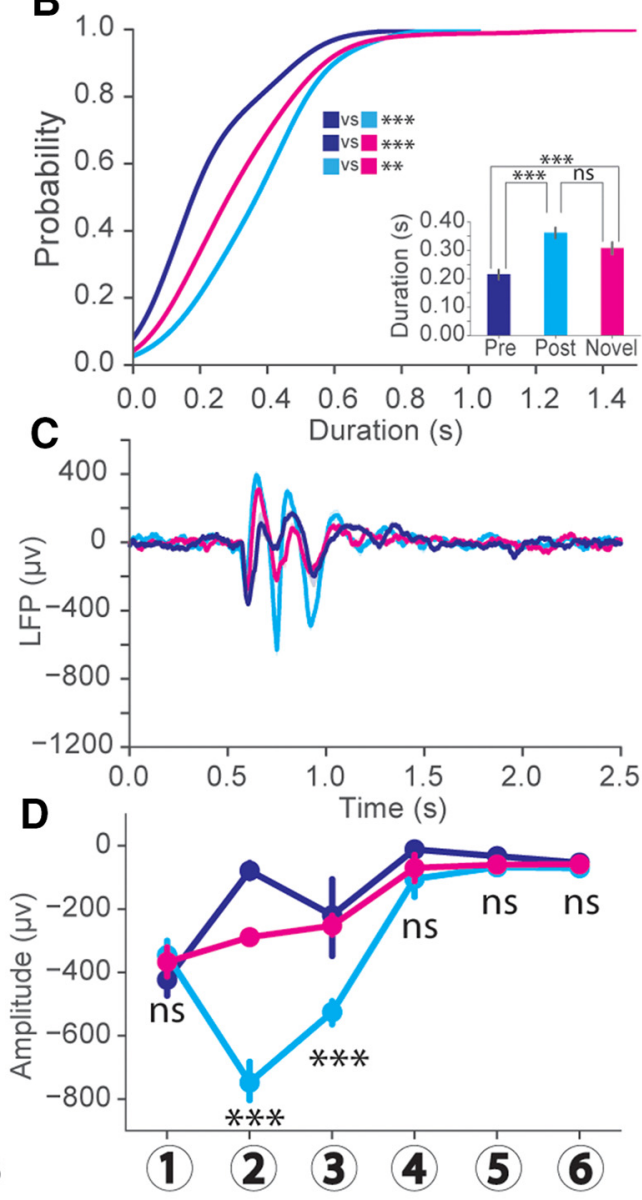

Figure 6. Experience-dependent oscillations in V1 are partially orientation selective. $\boldsymbol{A}, \boldsymbol{z}$ score of firing rate for all visually excited units from mice trained to a 200 ms drifting grating stimulus $(\mathrm{SF}=0.03 \mathrm{cpd}$ ) oriented 150 degrees after training (cyan). These mice were also shown a stimulus with the same SF, but oriented at 300 degrees (magenta). Line plots represent a population $z$ score (baseline normalized) for each condition. $\boldsymbol{B}, \mathrm{CDF}$ of oscillation duration: two-sample Kolmogorov-Smirnov test, blue versus cyan: $\mathrm{D}_{(150)}=0.58,3.42 \mathrm{E}-12 ;$ blue versus magenta: $\mathrm{D}_{(161)}=0.36, p=$ 2.79E-5; cyan versus magenta: $\mathrm{D}_{(157)}=0.25, p=0.07 \mathrm{E}-1$. Inset, Mean oscillation duration, Welch's unequal variances $t$ test, blue versus cyan: $t_{(150)}=5.69, p=6.33 \mathrm{E}-8$; blue versus magenta, $t_{(161)}=3.43, p=0.07 E-2$; cyan versus magenta: $t_{(157)}=1.87, p=0.06$ (units after peak detection): $N=74$ (cyan), $N=85$ (magenta), across 4 mice. $C$, Averaged traces of VEPs from the recordings shown in A.D, Bottom, Average amplitude of the VEPs at timings corresponding to 6 oscillation cycles. Two-way ANOVA (post-training only): (Factor 1: stimulus orientation; Factor 2: oscillation cycle) significant interaction between orientation and oscillation cycle on VEP amplitude $\left(F_{(5,132)}=51.69 ; p<0.001\right)$. Tukey HSD multiple comparison of means, 150 degree orientation (cyan) versus 300 degree orientation (magenta). Selected comparisons: Cycle1: $p>0.99$ (not significant). Cycle2: $p<0.001$. Cycle3: $p<0.001$. Cycle4: $p=0.08$ (not significant). Cycle5: $p>0.99$ (not significant). Cycle6: $p>0.99$ (not significant). $N=12$ trial averaged VEPs at 150 degree orientation, 12 trial averaged VEPs at 300 degree orientation across 4 mice. Error bars indicate SEM. ${ }^{* *} p<0.01,{ }^{* * *} p<$ 0.001 .

mice trained to $0.04 \mathrm{cpd}$ and for mice trained to $0.14 \mathrm{cpd}$ ) (Fig. $10 B)$. We then sought to determine how single cells respond to the trained versus novel stimuli. To do this, we created scatter plots that summarize how the duration of single-unit oscillatory activity varied depending on the stimulus SF (Fig. $10 C$, after SF = 0.04 training; and Fig. $10 D$, after $S F=0.14$ training). The duration of oscillations was quantified for each individual cell in response to the different stimuli, with pairwise relationships of familiar versus novel stimuli shown in the scatter plots. Each dot indicates a single unit, with the $x$-coordinate being the oscillation duration to familiar stimuli and the $y$-coordinate being the duration to novel stimuli of a particular SF. The red dashed line indicates a perfect correlation, corresponding to units responding the same way to the familiar or novel stimuli. If the majority of dots fall below the red line, single neurons responded with longer oscillatory activity to the familiar stimulus, whereas dots above line show that single units preferred novel stimuli. Black and green contours outline the density of the data points. This analysis demonstrated that single cells differentiated between distinct SF bands as evidenced by clustering toward the familiar stimulus (Fig. 10C). Among animals trained to $0.14 \mathrm{cpd}$, the difference between durations elicited by the trained versus novel stimuli was not as significant as after $\mathrm{SF}=0.04$ training (Fig. 10D). However, in both conditions, the strength of the familiarity-evoked oscillations was dependent on how close in SF the novel stimulus was to the trained one.

\section{Ongoing oscillations influence incoming visually evoked responses}

To determine the role of oscillatory activity in sensory information processing, we tested how an ongoing oscillation might influence the processing of subsequent visual stimuli. LFPs represent summed synchronized synaptic potentials in the local circuitry. Therefore, an ongoing oscillation may alter an incoming visually evoked response via summation of graded potentials, depending on the phase of the oscillation. To test this hypothesis, we presented visual stimuli at the high excitability $\theta$ phase (referred to here as "in phase") or the low excitability $\theta$ phase (re- 

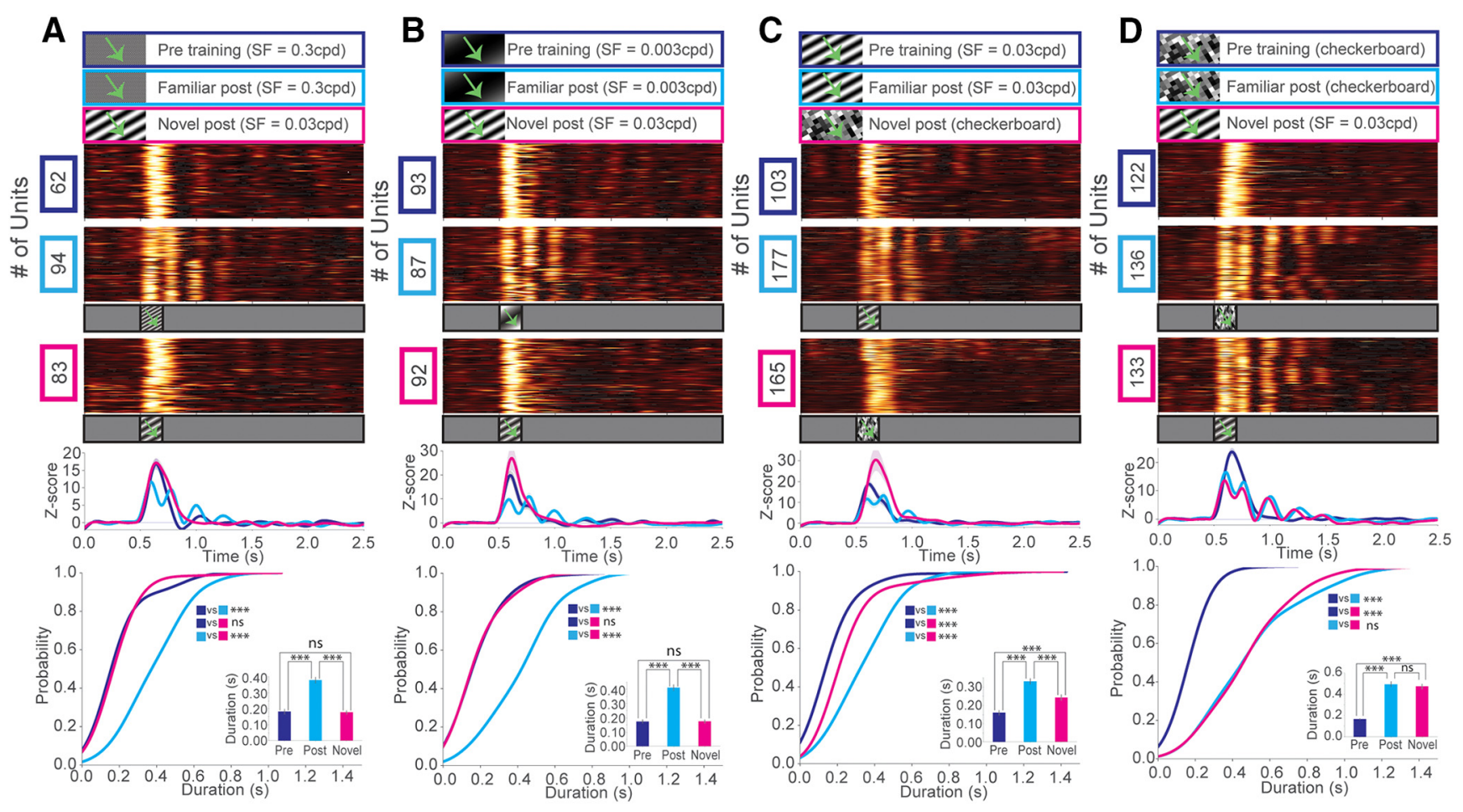

Figure 7. Experience-dependent oscillations in V1 are sensitive to the SF content of visual stimuli. $A, z$ scores of firing rates for mice shown a high $S F(S F=0.3 \mathrm{cpd})$ stimulus before training (blue) and after training (cyan). After training to the high SF stimulus, these mice were also shown an intermediate SF ( $0.03 \mathrm{cpd}$ ) stimulus for the first time (magenta). Line plots represent a population $z$ score (baseline normalized) for each condition. CDF of oscillation duration: two-sample Kolmogorov-Smirnov test, blue versus cyan: $D_{(132)}=0.77, p=3.74 \mathrm{E}-18$, blue versus magenta: $D_{(121)}=$ $0.22, p=0.071$, cyan versus magenta, $\mathrm{D}_{(142)}=0.72, p=1.97 \mathrm{E}-17$. Inset, Mean oscillation duration Welch's unequal variances $t$ test, blue versus cyan: $t_{(132)}=7.73, p=2.41 \mathrm{E}-12$, blue versus magenta: $t_{(121)}=0.18, p=0.851$, cyan versus magenta: $t_{(142)}=8.97, p=3.21 \mathrm{E}-15$ (units after peak detection: $N=56$ (blue), $N=78$ (cyan), $N=67$ (magenta) across 2 mice). $B$, Mice shown a low SF ( $0.003 \mathrm{cpd}$ ) stimulus before training (blue) and after training (cyan). After training to the low SF stimulus, these mice were also shown an intermediate SF (0.03 cpd) stimulus for the first time (magenta). CDF of oscillation duration: two-sample Kolmogorov-Smirnov test, blue versus cyan: $D_{(153)}=0.60, p=3.25 \mathrm{E}-13$, blue versus magenta: $D_{(171)}=0.11, p=0.56$, cyan versus magenta: $D_{(150)}=0.64, p=1.86 \mathrm{E}-14$. Inset, Bar graph of mean oscillation duration represents Welch's unequal variances $t$ test, blue versus cyan: $t_{(153)}=9.02, p=1.05 \mathrm{E}-14$, blue versus magenta: $t_{(171)}=0.08, p=0.931$, cyan versus magenta: $t_{(150)}=8.93, p=1.59 \mathrm{E}-14$ (units after peak detection: $N=88$ (blue), $N=67$ (cyan), $N=85$ (magenta) across 3 mice). $C$, Mice shown an intermediate SF $(0.03 \mathrm{cpd})$ stimulus before training (blue) and after training (cyan). After training to this stimulus, these mice were also shown a complex checkerboard stimulus for the first time (magenta). CDF of oscillation duration: two-sample Kolmogorov-Smirnov test, blue versus cyan: $D_{(203)}=0.66, p=5.04 \mathrm{E}-21$, blue versus magenta: $\mathrm{D}_{(203)}=0.53, p=9.57 \mathrm{E}-14$, cyan versus magenta: $D_{(218)}=0.47, p=1.71 \mathrm{E}-11$. Inset, Bar graph of mean oscillation duration represents Welch's unequal variances $t$ test, blue versus cyan: $t_{(203)}=8.13, p=4.18 \mathrm{E}-14$, blue versus magenta: $t_{(203)}=3.80, p=0.01 \mathrm{E}-2$, cyan versus magenta: $t_{(218)}=3.97, p=0.01 \mathrm{E}-2$ (units after peak detection): $N=95$ (blue), $N=110$ (cyan), $N=110$ (magenta) across $N=4$ mice. $D$, Mice shown a complex checkerboard stimulus before training (blue) and after training (cyan). After training to this stimulus, these mice were also shown an intermediate $S F(0.03 \mathrm{cpd})$ drifting grating stimulus for the first time (magenta). CDF of oscillation duration: two-sample Kolmogorov-Smirnov test, blue versus cyan: $\mathrm{D}_{(215)}=0.84, p=4.29 \mathrm{E}-35$, blue versus magenta: $\mathrm{D}_{(109)}=0.83, p=7.79 \mathrm{E}-34$, cyan versus magenta:, $\mathrm{D}_{(220)}=0.14, p=0.174$. Inset, Bar graph of mean oscillation duration represents Welch's unequal variances $t$ test, blue versus cyan: $t_{(215)}=12.4, p=1.93 \mathrm{E}-23$, blue versus magenta: $t_{(109)}=13.1, p=7.42 \mathrm{E}-25$, cyan versus magenta: $t_{(220)}=0.58, p=0.56$ (units after peak detection): $N=103$ (blue), $N=114$ (cyan), $N=108$ (magenta) across $N=4$ mice. Error bars indicate SEM. ${ }^{* *} p<0.001$.

ferred to here as "out of phase") relative to an ongoing visually evoked oscillation. We first recorded visually evoked responses to a single familiar stimulus after training to confirm the presence of $\theta$ oscillations. A second stimulus was then presented to evoke a response in phase with the third cycle of the oscillation, or to evoke a response out of phase with the third cycle. Interestingly, presentation of a visual stimulus in phase with the third cycle elicited a response that was approximately twice the amplitude of the first or third cycles elicited by a single stimulus. This increase did not occur when the second stimulus was presented out of phase (Fig. $11 A, B$ ). To estimate the translaminar currents underlying this phenomenon, we performed CSD analysis using trial averaged LFPs recorded across the cortical depth (Mitzdorf, 1985; Aizenman et al., 1996; Pettersen et al., 2006; Leski et al., 2007). Both current sinks and sources were stronger when the second stimulus was presented in phase with the third cycle compared with out of phase (Fig. 11C). This CSD analysis confirmed our VEP potentiation results.

\section{Oscillatory activity and pupil dynamics}

Pupil dilations are well known to report surprise, arousal, or cognitive load. Increases in pupil size have also been linked to a decrease in the power of spontaneous low-frequency oscillations in LFP recordings (Reimer et al., 2014; Vinck et al., 2015). To explore pupil dynamics and their relationship to visually evoked LFP oscillations, we simultaneously recorded changes in pupil size before and after training in response to grating stimuli ( $\mathrm{SF}=0.03$ cpd) or to checkerboard stimuli (novel stimuli) together with electrophysiological recordings. Time-frequency analysis of layer 4 VEPs recorded under these conditions revealed that novel stimuli elicit lower-power oscillatory activity beyond the stimulus presentation, similar to the pretraining condition (Fig. 12A,B). Pupil tracking of these animals revealed sustained increases in pupil size when the presented stimulus was novel (Fig. 12C,D). Importantly, this effect occurred both when an animal viewed a visual stimulus for the first time (the pretraining condition) and when a novel stimulus was presented after familiarity to a different stim- 

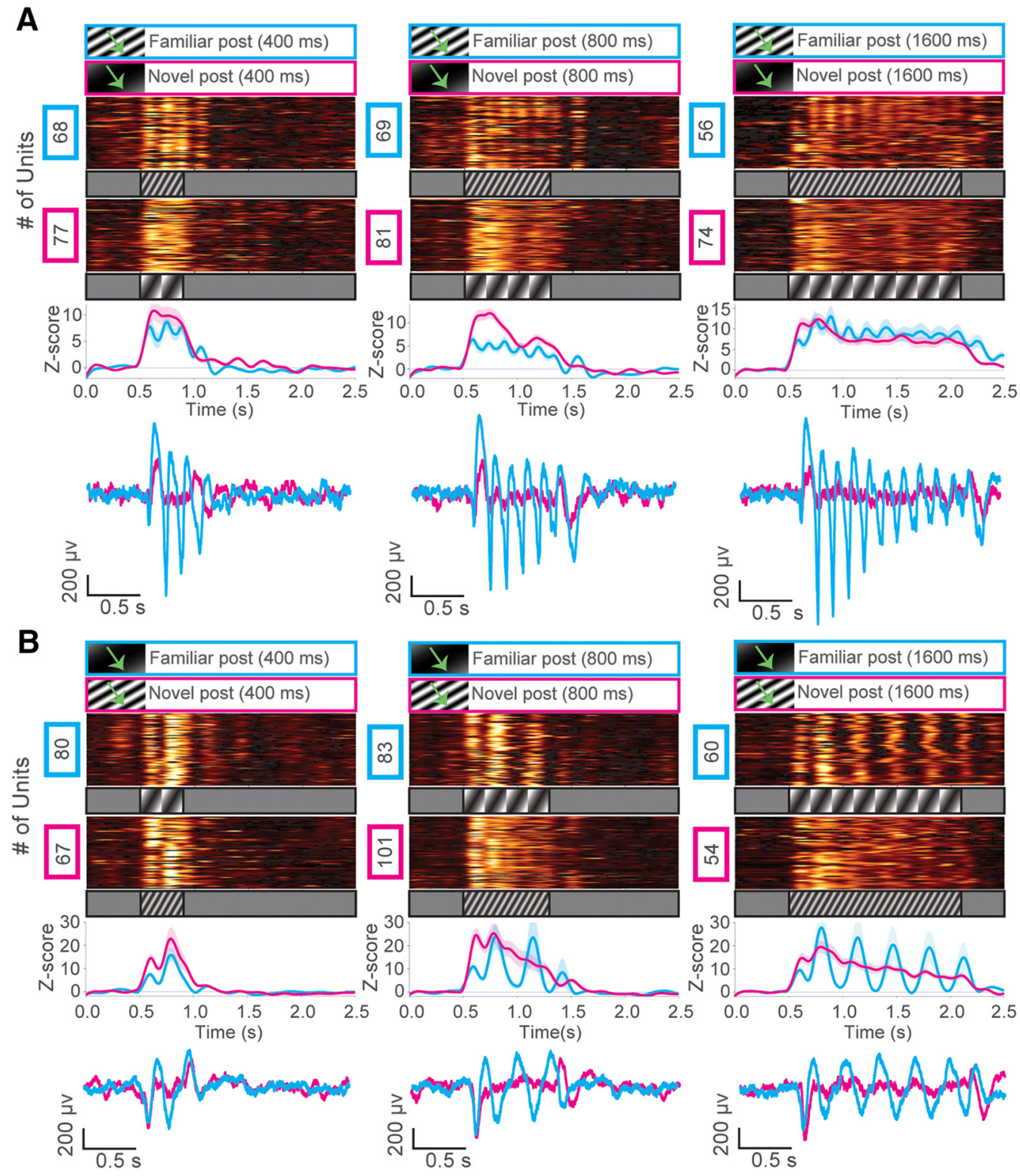

Figure 8. Oscillations extend with increasing durations of familiar visual stimuli. $A$, Top, zscore of firing rate for all visually excited units from mice trained to a $200 \mathrm{~ms}$ drifting grating low SF ( 0.003 cpd) stimulus after training (cyan) at $400 \mathrm{~ms}, 800 \mathrm{~ms}$, or $1600 \mathrm{~ms}$ durations ( $N=2$ mice). Unit oscillations extended across the entire duration of the stimulus but were not seen when a novel stimulus (magenta) with an intermediate SF $(0.03 \mathrm{cpd}$ ) was presented. Line plots represent a population z score (baseline normalized) for each condition. Bottom, Representative VEPs at each stimulus duration for novel and familiar stimuli. $B, z$ score of firing rate for all visually excited units from mice trained to a $200 \mathrm{~ms}$ drifting grating stimulus (SF $=0.03 \mathrm{cpd}$ ) after training (cyan) at $400 \mathrm{~ms}, 800$ $\mathrm{ms}$, or $1600 \mathrm{~ms}$ durations ( $N=2$ mice). Unit oscillations extended across the entire duration of the stimulus but were not seen when a novel stimulus (magenta) with a low SF ( $0.003 \mathrm{cpd}$ ) was presented. Line plots represent a population z score (baseline normalized) for each condition. Bottom, Representative VEPs at each stimulus duration for novel and familiar stimuli.

ulus was already established (the post-training novel condition). This response was not observed when animals were familiar with the presented stimulus, suggesting that these sustained pupil increases are representative of a surprise response. To explore the relationship between the power of oscillatory activity in LFPs and this surprise response, mean low-frequency $(4-30 \mathrm{~Hz})$ persistent (after stimulus presentation, $0.7-1.2 \mathrm{~s}$ ) oscillatory power was plotted against the mean pupil area percentage change of the surprise response for each individual mouse for which we successfully recorded both VEPs and pupil size. This analysis revealed that recordings with low oscillatory power (pretraining and novel conditions) correlated with higher surprise responses. The opposite was true for trained animals, where higher-power oscillations negatively correlated with surprise responses (Fig. 12E). Interestingly, similarly to the low-frequency oscillations, the power of high-frequency $(30-70 \mathrm{~Hz})$ persistent oscillatory activity also negatively correlated with the surprise response (Fig. 12F). These results suggest that these stimulus-evoked sustained pupil dilations depend on the perceptual novelty of the stimulus and are not solely mediated by the brain state changes traditionally associated with these types of pupil dynamics.

\section{Discussion}

Here, we determined the conditions and underlying mechanisms through which visually evoked low-frequency oscillations manifest in rodent primary visual cortex (V1). We have discovered that, after exposing mice daily to repetitively presented salient visual stimuli, persistent low-frequency oscillations emerge in both VEPs and individual units in V1. Principally, we show that (1) these oscillations arise in an experience-dependent manner; 
A

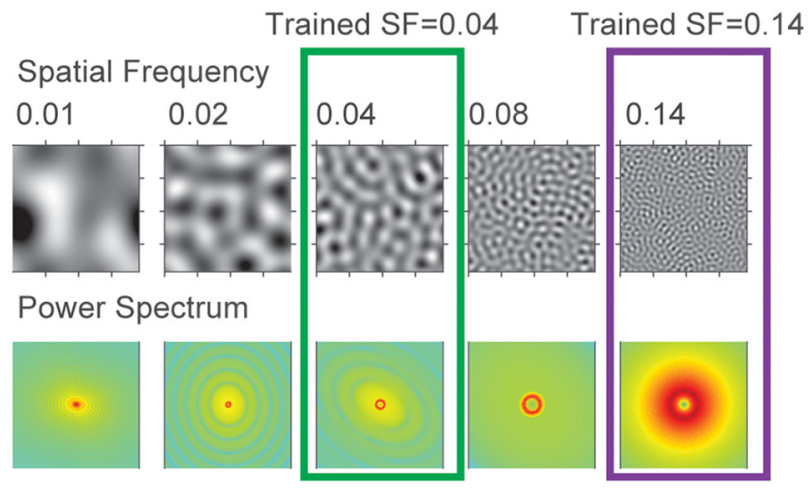

B

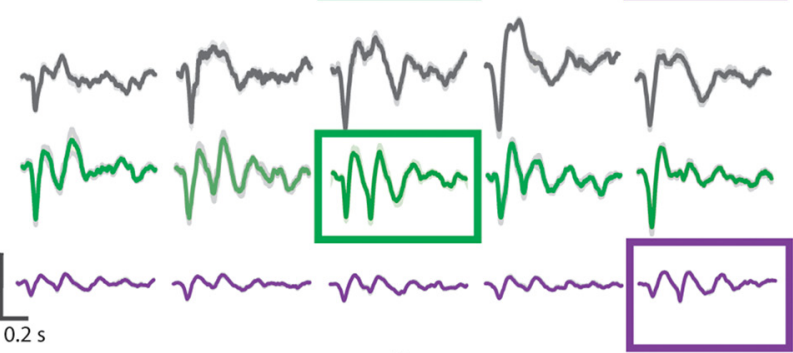

\section{D}
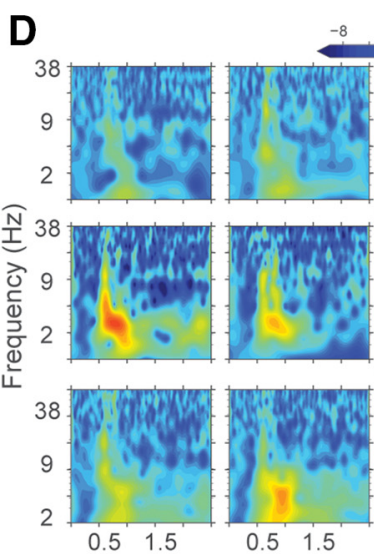

\begin{tabular}{ccc}
$\mathrm{dB}$ & 16 & 24 \\
8 & 16 & -2 \\
\hline
\end{tabular}

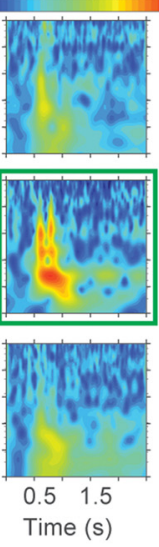

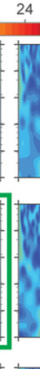
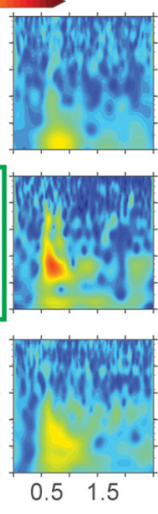

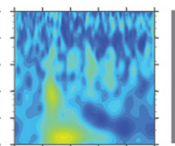

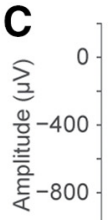

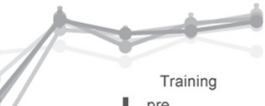

I pre

post $\mathrm{SF}=0.04$

post $\mathrm{SF}=0.14$
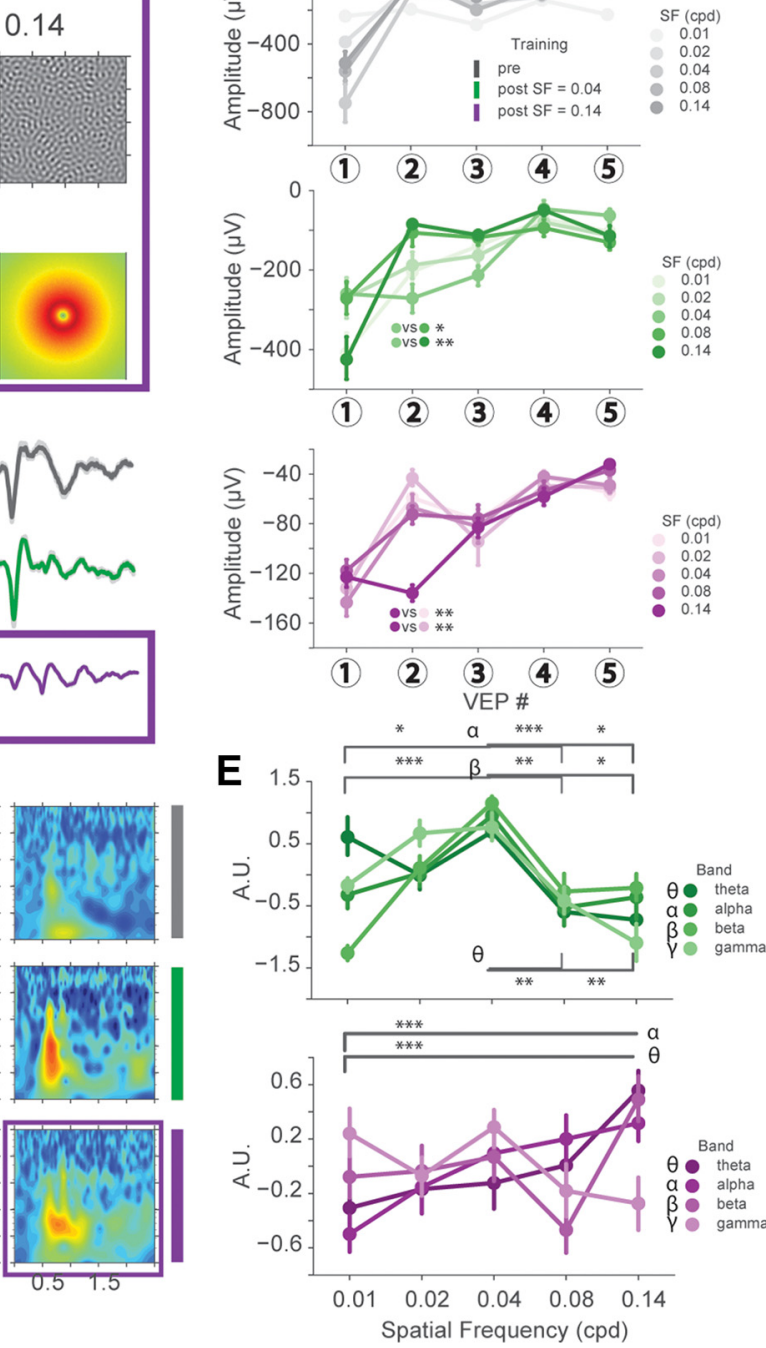

Figure 9. SF filtered noise visual stimuli dynamically modulates the power of oscillatory activity in LFPs in a SF-dependent manner. A, Top, Images of the SF filtered noise stimuli used. Bottom, Power spectrums demonstrating power distributions in the frequency domain. $\boldsymbol{B}$, Average VEP traces from all conditions showing that oscillatory activity emerges after training to stimuli with uniform narrowband SF. VEPs to the trained stimuli highlighted in the boxes. C, Potentiation of the second peak in VEP traces to the experienced but not novel stimuli is observed in both conditions. Significant interaction between the peak number and SF was found after $\mathrm{SF}=0.04$ and SF $=0.14$ training. Post $S F=0.04$ (green): two-way ANOVASF $\times$ VEP, $F_{(16,225)}=3.69(p<0.0001)$, post hoc Tukey test, $\mathrm{SF}=0.04$ versus $\mathrm{SF}=0.08(p=0.0185)$, versus $\mathrm{SF}=0.14(p=0.0064)$, versus $\mathrm{SF}=0.01$ (not significant), and $\mathrm{SF}=0.02$ (not significant), $n=12,11,8,10$, respectively; post $\mathrm{SF}=0.14$ (purple): two-way ANOVA, $S F \times$ VEP interaction, $F_{(16,405)}=3.02(p<0.0001)$, post hoc Tukey test, $S F=0.14$ versus $S F=0.01(p=0.0089)$, versus $S F=0.02(p=0.0023)$, versus $S F=0.04$ (not significant), and $S F=0.08$ (not significant), $n=18,17,15,18,18$, respectively. $\boldsymbol{D}$, The emergence of oscillatory activity in different frequency bands after animals experience visual stimuli can be observed in power spectra obtained from VEPs (from $\boldsymbol{B})$. The power of these oscillations was dynamically modulated in response to experienced versus novel stimuli. $\boldsymbol{E}$, Quantification of four frequency bands in point plots shows that the power of $\theta(4-8 \mathrm{~Hz}), \alpha(8-12 \mathrm{~Hz})$, and $\beta(12-25 \mathrm{~Hz})$ oscillations changed as a function of how close the SF of the presented stimulus was to that of the experienced stimulus. $S F=0.04$ (green): two-way ANOVA frequency band versus $S F, F_{(12,180)}=3.112738(p=0.0005)$, post hoc Tukey test: $\theta S F=0.04$ versus $S F=0.08(p=0.0022)$, versus $S F=0.14(p=0.001) ; \alpha: S F=0.04$ versus $S F=0.01(p=0.0324)$, versus $S F=0.08(p=0.0007)$, versus $S F=0.14(p=0.0138) ; \beta S F=0.04$ versus $S F=0.01(p<0.0001)$, versus $\mathrm{SF}=0.08(p=0.0041)$, versus $S F=0.14(0.0197) ; \mathrm{SF}=0.14$ (purple): two-way ANOVA frequency band versus $S F, F_{(12,324)}=4.485130(p<0.0001), \theta: S F=0.14$ versus $S F=0.01(p<$ $0.0001) ; \alpha: S F=0.14$ versus $S F=0.01(p<0.0001)$. Error bars indicate SEM. ${ }^{*} p<0.05,{ }^{* *} p<0.01,{ }^{* *} p<0.001$.

(2) they are sensitive to the familiarity of an animal to the SF content of a visual stimulus; (3) muscarinic receptors are necessary for both the induction and expression of these oscillations; and (4) ongoing oscillations directly influence the VEP amplitudes of oncoming stimuli depending on the $\theta$ excitability phase of the oscillation at which the oncoming stimuli are presented.

\section{Feature specificity of visual familiarity}

The brain continuously receives visual information and can automatically detect even minor changes in the visual environment. These statistical irregularities violate expectations generated by previous familiar stimuli. Indeed, object-based irregularities are automatically identified by the visual system, including changes in the geometric patterns or in the complex attributes of the natural stimuli (Stefanics et al., 2011; Stefanics and Czigler, 2012; Müller et al., 2013). Identification of these "surprising" stimuli may be based on both the long-term and short-term history of previous events. Surprising events carry novel information that can potentially be critical for survival. This process is necessary to continuously update the internal model of the world, which represents what the brain expects to see as "normal." However, "surprise" can only be detected whether familiarity has been 
A
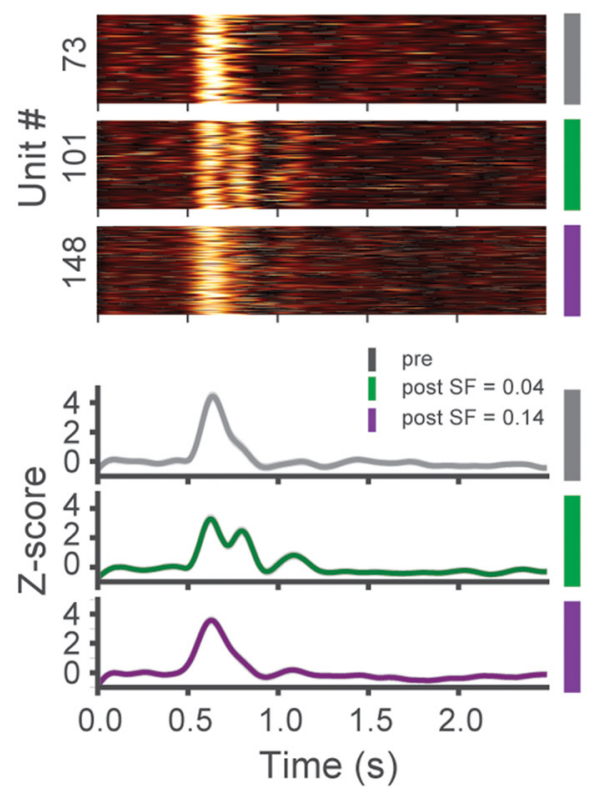

C

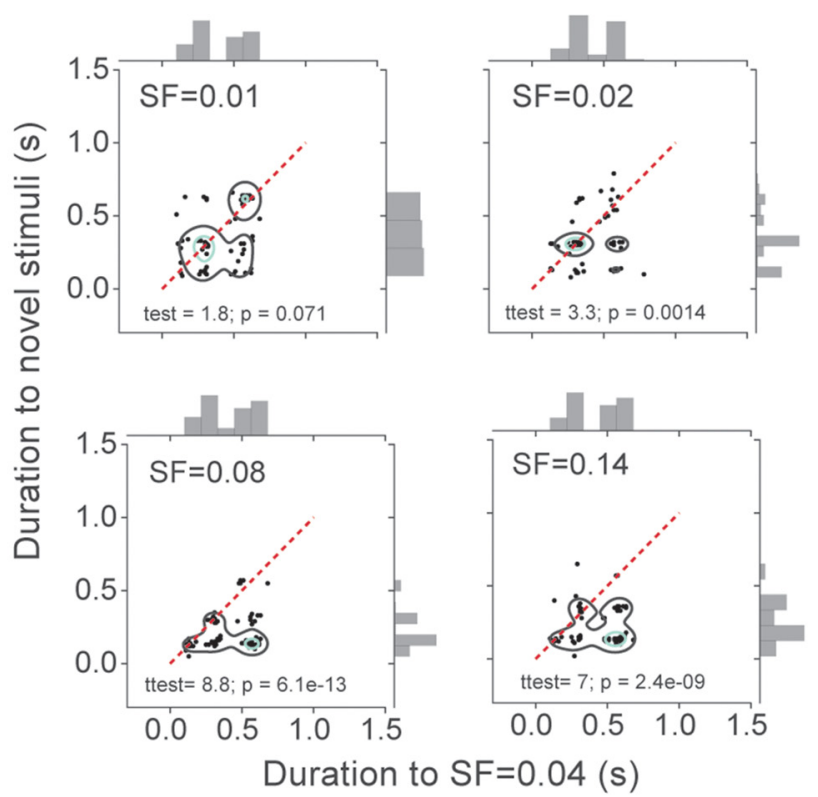

$\mathrm{SF}=0.14$
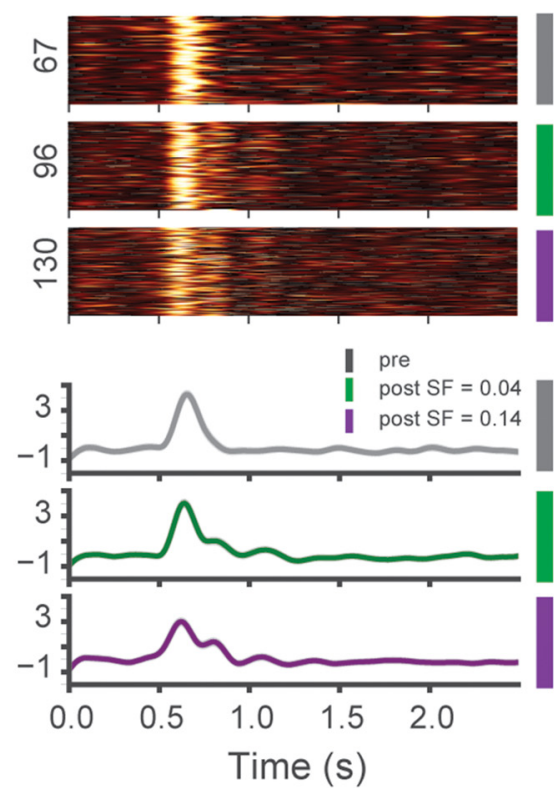

D
B
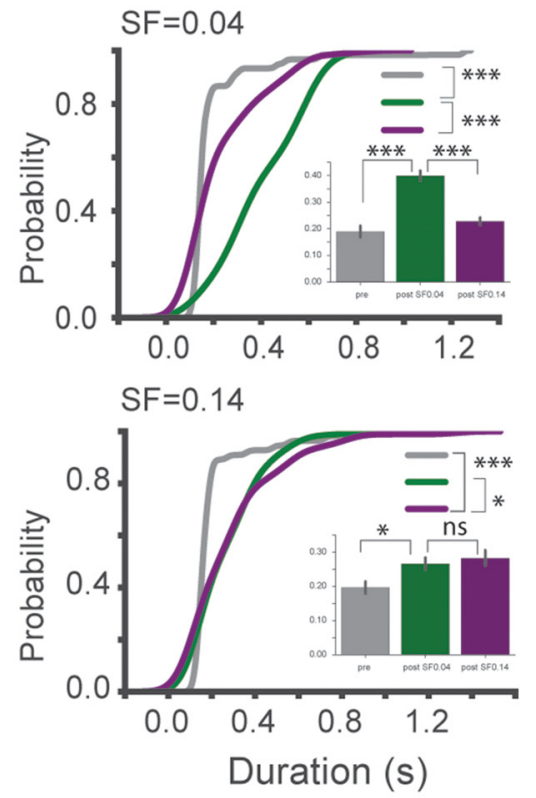

Figure 10. SF filtered noise stimuli dynamically modulate the duration of single-unit oscillatory activity in a SF-dependent manner. $A, 0$ scillatory activity emerges in single units after experiencing SF filtered noise visual stimuli and specific to the trained stimulus as shown in heat maps of zscored PSTHs: pre (gray); post SF $=0.04$ (green); post SF $=0.14$ (purple). Top left, Response to SF $=0.04$ across three different conditions. Top right, Same as left but for $S F=0.14$. Bottom left $z$ zscore time course of population average of the units depicted in heat maps. The emergence of oscillatory activity to SF $=0.04 \mathrm{after}$ experiencing that stimuli is seen, but not before or after $S F=0.14$ training. Bottom right, Same as bottom left but for $S F=0.14$, oscillations only observed after $S F=0.14$ training but not before/after $S F=0.04$ training. $B$, Cumulative distribution plots of the duration of oscillations in single units show significantly longer duration to the experienced stimulus compared with the novel or pretraining conditions; $\mathrm{SF}=0.04$ (top): two-sample Kolmogorov-Smirnov test post $S F=0.04$ training versus pre $(D=0.71, p=4.54 \mathrm{E}-16)$, versus $S F=0.14$ training $(D=0.52, p=3.02 \mathrm{E}-12)$. Inset, $t_{(139)}=7.16, p=4.21 \mathrm{E}-14, t_{(203)}=7.14, p=$ $1.61 \mathrm{E}-11, n=81,60,124$ units, respectively; $\mathrm{SF}=0.14$ (bottom): two-sample Kolmogorov-Smirnovtestpost $\mathrm{SF}=0.14$ training versus pre $(\mathrm{D}=0.41, p=9.25 \mathrm{E}-6)$, versus $\mathrm{SF}=0.04$ training $(\mathrm{D}=0.21, p=$ 0.03). Inset, $t$ test: $\left(t_{(145)}=2.57, p=0.01\right)$ and ( $t_{(171)}=-0.53, p=0.59$ (not significant), $n=92,55,81$, respectively. $C$, Duration of oscillatory activity in single units was significantly modulated by $S F$ of the stimulus as evident from scatter plots (red dashed line indicates perfect correlation between conditions). Each dot indicates duration of single neuron's oscillatory activity to the familiar ( $x$-axis) versus novel $\left(y\right.$-axis) stimuli, so that clustering of dots below red line shows preference to the familiar stimulus. Green and black contours outline KDE of dots. Paired $t$ test:SF $=0.04$ versus SF $=0.01\left(t_{(59)}=1.8, p=0.071\right)$, versus $\mathrm{SF}=0.02\left(t_{(68)}=3.3, p=0.0014\right)$, versus $\mathrm{SF}=0.08\left(t_{(69)}=8.8, p=6.1 \mathrm{E}-13\right)$, versus $\mathrm{SF}=0.14\left(t_{(59)}=7, p=2.4 \mathrm{E}-9\right), n=60,69,70,60$, respectively. $D$, Same as $D$ but for animals trained to $\mathrm{SF}=$ $0.14 . S F=0.14$ versus $S F=0.01\left(t_{(54)}=2.3, p=0.024\right)$, versus $S F=0.02\left(t_{(68)}=2.1, p=0.037\right)$, versus $S F=0.04\left(t_{(79)}=1.9, p=0.057\right)$, versus $S F=0.08\left(t_{(79)}=-0.13, p=0.9\right.$ (not significant), $n=55,69,80,80$, respectively. Error bars indicate SEM. ${ }^{*} p<0.05,{ }^{* * *} p<0.001$.

established toward at least some visual stimuli, and the corresponding predictions based on these stimuli have been generated. Extraction of the common statistical features of the repeating visual stimuli may serve as an automatic "perceptual filter" (Ste- fanics et al., 2014). Our results are consistent with this theory. Repeated presentation of the visual stimuli of a particular SF leads to the development of familiarity to its SF mediated by the lowfrequency oscillations. Although we did not observe the all-or- 
1 stimulus only

second stimulus, presented in phase with cycle 3

second stimulus, presented out of phase with cycle 3

A

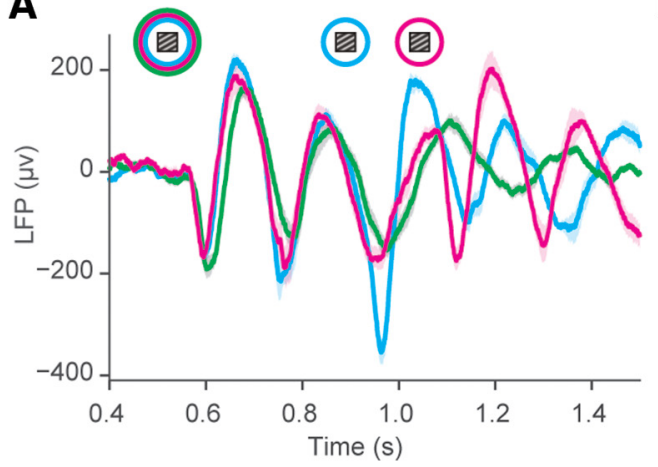

B

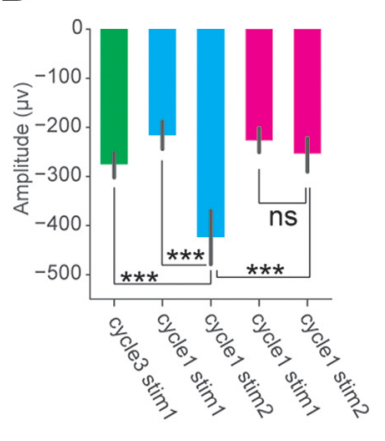

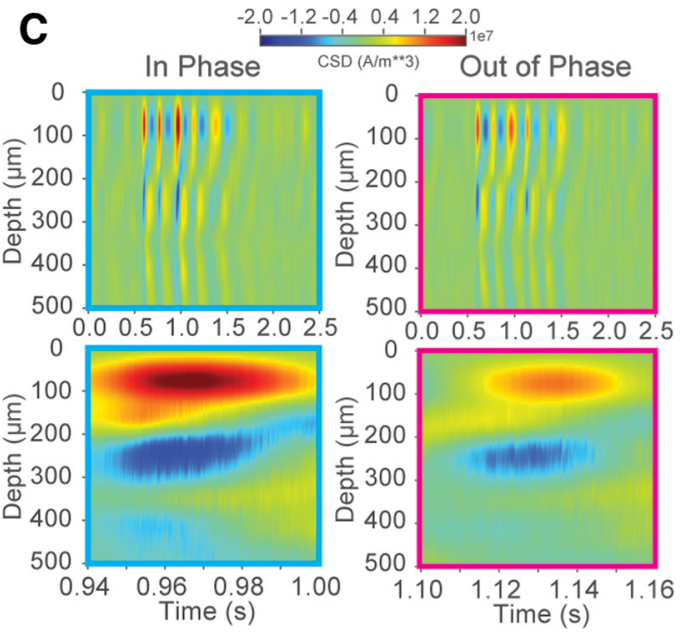

Figure 11. Ongoing $\theta$ oscillations influence incoming visually evoked responses. $\boldsymbol{A}$, Averaged VEPs after training when a single familiar stimulus is presented (green trace) or when a second familiar stimulus is presented at a delay either in phase (cyan trace) or out of phase (magenta trace) with the third cycle of the oscillation. B, Average amplitude of the first cycle VEP for the first stimulus (Cycle1, stim1) presented or the second stimulus (Cycle1, stim2) when it is presented either in phase or out of phase with the third cycle of the oscillation. An additional comparison is made between the average amplitude at the third cycle elicited by the 1 stimulus control and the average amplitude of the first cycle for the second stimulus presented in phase or out of phase. Two-way ANOVA: Factor 1, visual stimulus condition (in phase familiar or out of phase familiar); Factor 2, oscillation cycle (cycle1 stim1 or cycle1 stim 2). There was a significant interaction between visual stimulus condition and oscillation cycle on VEP amplitude $\left(F_{(1,68)}=20.5\right)(p<0.0001)$. Tukey HSD multiple comparison of means, selected comparisons: IN_stim1_cycle1_familiar versus IN_stim2_cycle1_familiar, $p<0.0001$. 0UT_stim1_cycle1_familiar versus 0UT_stim2_cycle1_familiar, $p=0.77$ (not significant). IN_stim2_cycle1_familiar versus 0UT_stim2_cycle1_familiar, $p<$ 0.0001. Welch's two-sample $t$ tests: CTRL_stim1_cycle1_familiar versus IN_stim2_cycle1_familiar, $p=1.20 \mathrm{E}-4$. CTRL_stim1_cycle1_familiar versus 0UT_stim2_cycle1_familiar, $p=0.355$ (not significant) ( $N=18$ trial averaged VEPs for each condition across 6 mice). $C$, Representative CSD analysis of oscillatory activity when the second stimulus is presented in phase (Top, cyan) or out of phase (Bottom, magenta) with the third cycle of the oscillation. Each example is zoomed in to show the current sink and source corresponding to the timing when the second stimulus is presented. Error bars indicate SEM. ${ }^{* * *} p<0.001$.

none oscillatory response to the familiar vs novel stimuli, this is not surprising given that individual neurons respond to a range of different SF. The power of the oscillatory activity in LFP and the response duration of single units dynamically varied based on how close the presented SF band was to the trained one. Significant differences were observed in both LFP and single units' activity when comparing the responses to the trained and the novel distant SF bands. Furthermore, we have demonstrated that the oscillations are not dependent on the choice of the stimulus by training to two different low and high SF bands. Using pure SF bands without confounding elements, such as orientation, we have demonstrated that the oscillatory activity was modulated as a function of the SF intrinsic to the familiar visual stimulus. We propose that this oscillatory code is a common strategy primary visual cortex employs for segregating familiar versus novel visual inputs.

\section{Oscillatory activity and arousal}

Locomotion exerts an excitatory influence on V1, pushing most neurons into a more depolarized state and causing significant increases in visually evoked firing rates (Niell and Stryker, 2010; Polack et al., 2013; Reimer et al., 2014). This effect is thought to be a gain modulation, as firing rates are increased without a loss in feature selectivity (Cardin et al., 2008; Niell and Stryker, 2010; Polack et al., 2013). Increases in pupil size are also a robust measure of arousal and are highly correlated with periods of locomotion. Interestingly, aroused states (characterized by periods of pupil dilation or locomotion) are correlated with a reduction in the power of low-frequency oscillations in LFPs or membrane potential recordings in V1 (Niell and Stryker, 2010; Reimer et al., 2014; Vinck et al., 2015). Consistent with these reports, we found that stimulus-evoked increases in pupil size were correlated with decreased low-frequency oscillatory $(4-30 \mathrm{~Hz})$ activity. These pupil size increases occurred under conditions when visual stim- uli were novel to the animal, indicating a surprise-based increase in arousal levels. We also observed significantly higher firing rates of stimulus-locked responses to novel stimuli compared with familiar stimuli, which conversely showed a decrease in stimulusevoked firing rates following perceptual training. This observation is consistent with the reports of an arousal-based gain modulation of visually evoked responses. It is also consistent with the previously reported stimulus-specific adaptation leading to a decrease in the peak stimulus-evoked firing rate following repeated exposure to the same stimulus (Hamm and Yuste, 2016; Vinken et al., 2017). On the other hand, we observed a sustained poststimulus decrease $(\sim 1 \%)$ in pupil size correlated with higherpower low-frequency oscillations. This observation is consistent with the previous reports that the peak amplitude of lowfrequency oscillatory activity is correlated with pupil constriction in awake mice (Reimer et al., 2014). However, the increase in low-frequency oscillatory activity we observed only occurred after visual stimulus presentation and was not spontaneous. Furthermore, we have also observed an increase in the power of high-frequency $\gamma$ oscillations following training, which are traditionally associated with the aroused cortical state and increased pupil size. This finding of higher-power $\gamma$ oscillations correlated with pupil constriction after training suggests that the low- and high-frequency sustained oscillations are representative of learningrelated changes in the cortex and not simply brain state alterations. The changes in pupil size may represent the influence of neuromodulators known to mediate arousal. Transient changes in pupil dilation are preceded by increases in noradrenergic and cholinergic activity, even in the absence of locomotion (Reimer et al., 2016). The gain modulatory effects often attributed to locomotion can also be influenced by direct cholinergic or noradrenergic manipulation, demonstrating that neuromodulation alone can exert some of these effects (Goard and Dan, 2009; Pinto et al., 2013; Polack et al., 2013). However, both noradrenergic and 


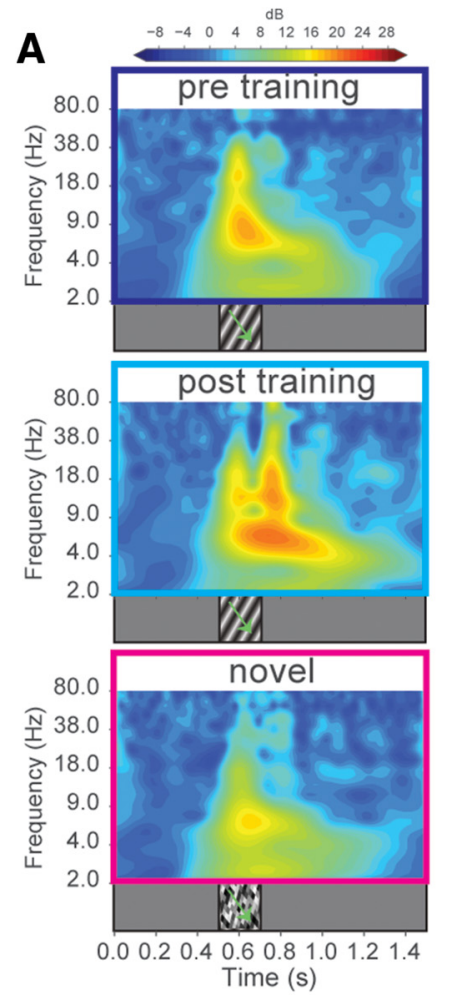

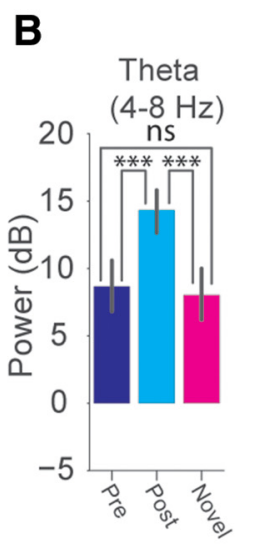

C
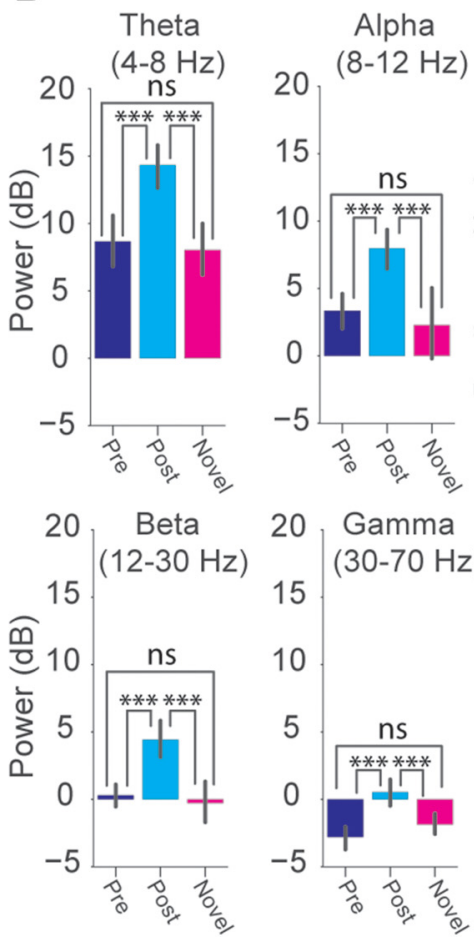

E

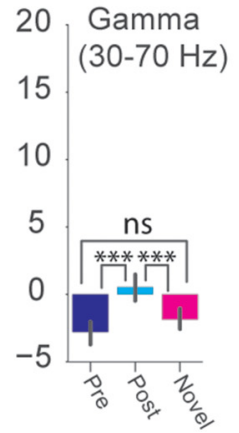

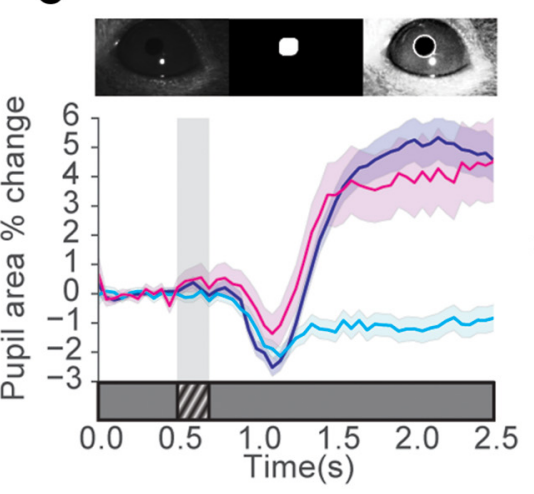

D

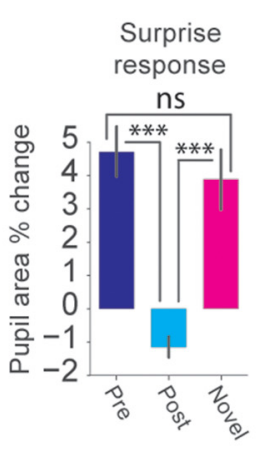

$\mathbf{F}$

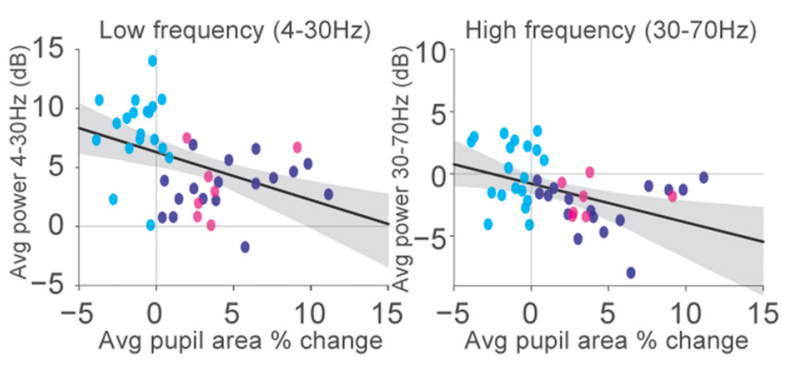

Figure 12. Transient pupil dynamics report familiarity to visual stimuli. $A$, Averaged time-frequency spectrograms of layer 4 VEP responses to single $200 \mathrm{~ms}$ sinusoidal drifting gratings pretraining (blue, from 24 mice), post-training (cyan, from 24 mice), and during the presentation of a novel (checkerboard) stimuli (magenta, from 10 mice). LFP data from Figures 1 and 7 ( were combined with recordings from 6 additional mice and plotted together. $\boldsymbol{B}$, The change in power (from baseline) across $\theta(4-8 \mathrm{~Hz}), \alpha(8-12 \mathrm{~Hz}), \beta(12-30 \mathrm{~Hz})$, and $\gamma(30-70 \mathrm{~Hz})$ frequency bands is shown pretraining (blue), post-training (cyan), and during novel stimuli (magenta). Welch's $t$ test, $\theta$ : blue versus cyan, $t_{(144)}=-4.48, p=4.94 \mathrm{E}-5 ;$ blue versus magenta, $t_{(100)}=0.44, p=0.66 ;$ cyan versus magenta, $t_{(100)}=4.72,6.13 \mathrm{E}-5 ; \alpha$ : blue versus cyan, $t_{(142)}=-4.46, p=5.27 \mathrm{E}-5$; blue versus magenta, $t_{(100)}=0.696, p=0.49 ;$ cyan versus magenta, $t_{(100)}=3.68, p=0.01 \mathrm{E}-1 ; \beta$ : blue versus cyan, $t_{(142)}=-4.82, p=2.21 \mathrm{E}-5$; blue versus magenta, $t_{(100)}=0.58, p=0.56$; cyan versus magenta, $t_{(100)}=4.21, p=2.0 \mathrm{E}-4 ; \gamma$ : blue versus cyan, $t_{(142)}=-4.93, p=1.16 \mathrm{E}-5$; blue versus magenta, $t_{(100)}=-1.53, p=0.13$; cyan versus magenta, $t_{(100)}=3.59, p=9 \mathrm{E}-4$. C, Top, Pupil detection and tracking. Bottom, Baseline normalized pupil area percentage change for pretraining (blue), post-training (cyan), and during novel stimulus presentation (magenta). $\boldsymbol{D}$, Averaged values of the mean pupil area percentage change during the surprise response period $\left(1.4-2.5 \mathrm{~s}\right.$ ). Surprise response: Welch's $t$ test, pre (blue) versus post familiar (cyan), $t_{(34)}=6.81, p=1 \mathrm{E}-06$, pre (blue) versus post novel (magenta): $t_{(22)}=0.67, p=0.5$. post familiar (cyan) versus post novel (magenta): $t_{(24)}=5.27, p=9.5 \mathrm{E}-04$. $\boldsymbol{E}$, Scatter plots of mean pupil area percentage change versus mean power of layer $4 \mathrm{LFP}$ low-frequency $(4-30 \mathrm{~Hz})$ or $(\boldsymbol{F})$ high-frequency $(30-70$ $\mathrm{Hz}$ ) oscillations. Each data point represents a recording from an individual mouse before training (blue) after training (cyan) and after training with the presentation of a novel stimulus (magenta). Only recordings where the pupil was successfully tracked are plotted: pre (blue): 17 of 24 mice; post (cyan): 19 of 24 mice; novel (magenta): 7 of 10 mice. A regression line is drawn across the datasets (low-frequency plot: regression coefficient $=-0.40 . p=5.05 \mathrm{E}-03$; high-frequency plot: regression coefficient $=-0.31, p=4.0 \mathrm{E}-3$ ). Error bars indicate $\mathrm{SEM}$. ${ }^{* * *} p<0.001$.

cholinergic systems are also involved in circuit and synaptic plasticity, which makes it difficult to disambiguate the role of neuromodulators in arousal and learning (Seol et al., 2007). Our results show that muscarinic receptors are necessary for both the induction and expression familiarity-evoked oscillations, consistent with other work highlighting the importance of the cholinergic system in the regulation of persistent activity or learning and memory (Chubykin et al., 2013; Gavornik and Bear, 2014; Vandecasteele et al., 2014). A detailed study of how neuromodulators can regulate arousal and oscillatory rhythms in V1 in the context of learning is one of the important future directions of our research.

\section{Oscillations and neural processing of visual familiarity}

Animals need to effectively segregate familiar from novel stimuli to successfully interpret the environment around them. There is a significant body of evidence demonstrating the differential processing of stimuli in sensory cortices based on previous experience (Gavornik and Bear, 2014; Cooke et al., 2015; Kato et al., 2015; Makino and Komiyama, 2015; Poort et al., 2015), which in some cases results in persistent activity beyond the stimulus presentation (Shuler and Bear, 2006; Zold and Hussain Shuler, 2015). However, neither the stimulus nor the visual feature specificity of experience-dependent persistent activity has been dem- onstrated. Here, we show, for the first time, a neural signature of visual familiarity in $\mathrm{V} 1$ that manifests as persistent low-frequency $(\theta, \alpha, \beta)$ oscillations after perceptual experience. Furthermore, we have shown that ongoing visually evoked oscillatory activity can influence the processing of incoming visual stimuli. Visual information arriving at the peak of the oscillation cycle can be amplified while information arriving at the trough may be unchanged or attenuated. There is evidence for a similar phenomenon in the auditory cortex, whereby a corticothalamic circuit can reset the phase of low-frequency $(\delta, \theta)$ rhythms to regulate the salience of auditory stimuli (Guo et al., 2017). These data and the work by others suggest that oscillations can filter or gate information flow in the brain (Schroeder and Lakatos, 2009). Our findings that familiarity is signaled by low-frequency oscillatory activity are well aligned with studies reporting the existence of different frequency channels supporting bottom-up and top-down modulation. It has been suggested by several studies that $\gamma$ oscillations are involved in feedforward information flow, whereas $\alpha$ and $\beta$ bands are involved in feedback (Buffalo et al., 2011; van Kerkoerle et al., 2014; Bastos et al., 2015; Michalareas et al., 2016). There is still some debate about the $\theta$ band, with different groups differentially reporting its influence on the directionality of information flow (Bastos et al., 2015; Michalareas et al., 2016). 
However, oscillations may also be important for information exchange and synchronization between different brain areas (Buzsáki and Draguhn, 2004; Fries, 2005). Consistent with this theory, $\theta$ synchronization between visual area $\mathrm{V} 4$ and prefrontal cortex has been reported to be necessary for the maintenance of visual short-term memories (Liebe et al., 2012). Although visual stimulus familiarity significantly upregulated all frequency bands tested beyond the time of visual stimulation, the most striking difference pretraining versus post-training lies in the strengthening of the $\alpha$ and $\beta$ bands. Interestingly, these increases are aligned with the potentiation of the second peak seen in the LFP, which is the most prominent signature of familiarity in our data. Furthermore, our experiment with pure SF bands demonstrated that $\alpha$ and $\beta$ (and to a lesser extent $\theta$ ) power are significantly modulated depending on how close the presented (SF) is from the trained stimulus. Top-down signals reporting familiarity might manifest through the emergence of these $\alpha$ and $\beta$ oscillations, although we also see an increase in the power of bottom-up $\gamma$ frequency band after training. Future studies are necessary to explore how these oscillations propagate across different cortical areas, and to dissect the molecular, cellular, and circuit mechanisms that drive them.

\section{References}

Aizenman CD, Kirkwood A, Bear MF (1996) A current source density analysis of evoked responses in slices of adult rat visual cortex: implications for the regulation of long-term potentiation. Cereb Cortex 6:751-758. CrossRef Medline

Bastos AM, Vezoli J, Bosman CA, Schoffelen JM, Oostenveld R, Dowdall JR, De Weerd P, Kennedy H, Fries P (2015) Visual areas exert feedforward and feedback influences through distinct frequency channels. Neuron 85:390-401. CrossRef Medline

Besedovsky L, Ngo HV, Dimitrov S, Gassenmaier C, Lehmann R, Born J (2017) Auditory closed-loop stimulation of EEG slow oscillations strengthens sleep and signs of its immune-supportive function. Nat Commun 8:1984. CrossRef Medline

Bigl V, Woolf NJ, Butcher LL (1982) Cholinergic projections from the basal forebrain to frontal, parietal, temporal, occipital, and cingulate cortices: a combined fluorescent tracer and acetylcholinesterase analysis. Brain Res Bull 8:727-749. CrossRef Medline

Buffalo EA, Fries P, Landman R, Buschman TJ, Desimone R (2011) Laminar differences in $\gamma$ and $\alpha$ coherence in the ventral stream. Proc Natl Acad Sci U S A 108:11262-11267. CrossRef Medline

Buschman TJ, Miller EK (2007) Top-down versus bottom-up control of attention in the prefrontal and posterior parietal cortices. Science 315: 1860-1862. CrossRef Medline

Buzsáki G, Draguhn A (2004) Neuronal oscillations in cortical networks. Science 304:1926-1929. CrossRef Medline

Cardin JA, Palmer LA, Contreras D (2008) Cellular mechanisms underlying stimulus-dependent gain modulation in primary visual cortex neurons in vivo. Neuron 59:150-160. CrossRef Medline

Chan RW, Leong AT, Ho LC, Gao PP, Wong EC, Dong CM, Wang X, He J, Chan YS, Lim LW, Wu EX (2017) Low-frequency hippocampal-cortical activity drives brain-wide resting-state functional MRI connectivity. Proc Natl Acad Sci U S A 114:E6972-E6981. CrossRef Medline

Chen G, Rasch MJ, Wang R, Zhang XH (2015) Experience-dependent emergence of beta and gamma band oscillations in the primary visual cortex during the critical period. Sci Rep 5:17847. CrossRef Medline

Chubykin AA, Roach EB, Bear MF, Shuler MG (2013) A cholinergic mechanism for reward timing within primary visual cortex. Neuron 77:723735. CrossRef Medline

Cohen MX (2014) Analyzing neural time series data. Cambridge, MA: Massachusetts Institute of Technology.

Cooke SF, Komorowski RW, Kaplan ES, Gavornik JP, Bear MF (2015) Visual recognition memory, manifested as long-term habituation, requires synaptic plasticity in V1. Nat Neurosci 18:262-271. CrossRef Medline

Durkin J, Suresh AK, Colbath J, Broussard C, Wu J, Zochowski M, Aton SJ (2017) Cortically coordinated NREM thalamocortical oscillations play an essential, instructive role in visual system plasticity. Proc Natl Acad Sci U S A 114:10485-10490. CrossRef Medline

Einstein MC, Polack PO, Tran DT, Golshani P (2017) Visually evoked 3-5 $\mathrm{Hz}$ membrane potential oscillations reduce the responsiveness of visual cortex neurons in awake behaving mice. J Neurosci 37:5084-5098. CrossRef Medline

Frenkel MY, Sawtell NB, Diogo AC, Yoon B, Neve RL, Bear MF (2006) Instructive effect of visual experience in mouse visual cortex. Neuron 51:339-349. CrossRef Medline

Fries P (2005) A mechanism for cognitive dynamics: neuronal communication through neuronal coherence. Trends Cogn Sci 9:474-480. CrossRef Medline

Friese U, Daume J, Göschl F, König P, Wang P, Engel AK (2016) Oscillatory brain activity during multisensory attention reflects activation, disinhibition, and cognitive control. Sci Rep 6:32775. CrossRef Medline

Gavornik JP, Bear MF (2014) Learned spatiotemporal sequence recognition and prediction in primary visual cortex. Nat Neurosci 17:732-737. CrossRef Medline

Goard M, Dan Y (2009) Basal forebrain activation enhances cortical coding of natural scenes. Nat Neurosci 12:1444-1449. CrossRef Medline

Gu Q (2003) Contribution of acetylcholine to visual cortex plasticity. Neurobiol Learn Mem 80:291-301. CrossRef Medline

Guo W, Clause AR, Barth-Maron A, Polley DB (2017) A corticothalamic circuit for dynamic switching between feature detection and discrimination. Neuron 95:180-194.e5. CrossRef Medline

Hamm JP, Yuste R (2016) Somatostatin interneurons control a key component of mismatch negativity in the mouse visual cortex. Cell Rep 16:597604. CrossRef Medline

Jensen O, Tesche CD (2002) Frontal $\theta$ activity in humans increases with memory load in a working memory task. Eur J Neurosci 15:1395-1399. CrossRef Medline

Kang JI, Huppé-Gourgues F, Vaucher E (2014) Boosting visual cortex function and plasticity with acetylcholine to enhance visual perception. Front Syst Neurosci 8:172. CrossRef Medline

Karalis N, Dejean C, Chaudun F, Khoder S, Rozeske RR, Wurtz H, Bagur S, Benchenane K, Sirota A, Courtin J, Herry C (2016) 4-Hz oscillations synchronize prefrontal-amygdala circuits during fear behavior. Nat Neurosci 19:605-612. CrossRef Medline

Kato HK, Gillet SN, Isaacson JS (2015) Flexible sensory representations in auditory cortex driven by behavioral relevance. Neuron 88:1027-1039. CrossRef Medline

Kim B, Kocsis B, Hwang E, Kim Y, Strecker RE, McCarley RW, Choi JH (2017) Differential modulation of global and local neural oscillations in REM sleep by homeostatic sleep regulation. Proc Natl Acad Sci U S A 114:E1727-E1736. CrossRef Medline

Lee H, Simpson GV, Logothetis NK, Rainer G (2005) Phase locking of single neuron activity to $\theta$ oscillations during working memory in monkey extrastriate visual cortex. Neuron 45:147-156. CrossRef Medline

Leski S, Wójcik DK, Tereszczuk J, Swiejkowski DA, Kublik E, Wróbel A (2007) Inverse current-source density method in 3D: reconstruction fidelity, boundary effects, and influence of distant sources. Neuroinformatics 5:207-222. CrossRef Medline

Liebe S, Hoerzer GM, Logothetis NK, Rainer G (2012) Theta coupling between $\mathrm{V} 4$ and prefrontal cortex predicts visual short-term memory performance. Nat Neurosci 15:456-462, S1-S2. CrossRef Medline

Makino H, Komiyama T (2015) Learning enhances the relative impact of top-down processing in the visual cortex. Nat Neurosci 18:1116-1122. CrossRef Medline

McCoy P, Norton TT, McMahon LL (2008) Layer 2/3 synapses in monocular and binocular regions of tree shrew visual cortex express $\mathrm{mAChR}$ dependent long-term depression and long-term potentiation. J Neurophysiol 100:336-345. CrossRef Medline

Mesulam MM, Mufson EJ, Levey AI, Wainer BH (1983) Cholinergic innervation of cortex by the basal forebrain: cytochemistry and cortical connections of the septal area, diagonal band nuclei, nucleus basalis (substantia innominata), and hypothalamus in the rhesus monkey. J Comp Neurol 214: 170-197. CrossRef Medline

Michalareas G, Vezoli J, van Pelt S, Schoffelen JM, Kennedy H, Fries P (2016) Alpha-beta and gamma rhythms subserve feedback and feedforward influences among human visual cortical areas. Neuron 89:384-397. CrossRef Medline

Mitzdorf U (1985) Current source-density method and application in cat 
cerebral cortex: investigation of evoked potentials and EEG phenomena. Physiol Rev 65:37-100. CrossRef Medline

Müller D, Widmann A, Schröger E (2013) Object-related regularities are processed automatically: evidence from the visual mismatch negativity. Front Hum Neurosci 7:259. CrossRef Medline

Mussel P, Ulrich N, Allen JJ, Osinsky R, Hewig J (2016) Patterns of $\theta$ oscillation reflect the neural basis of individual differences in epistemic motivation. Sci Rep 6:29245. CrossRef Medline

Niell CM, Stryker MP (2010) Modulation of visual responses by behavioral state in mouse visual cortex. Neuron 65:472-479. CrossRef Medline

Pachitariu M, Steinmetz NA, Kadir SN, Carandini M, Harris KD (2016) Fast and accurate spike sorting of high-channel count probes with KiloSort. In: Proceedings of the Advances in Neural Information Processing Systems 29. (Lee DD, Sugiyama M, Luxburg UV, Guyon I, Garnett R, eds), pp 4448-4456. New York, NY: Curran Associates, Inc.

Pettersen KH, Devor A, Ulbert I, Dale AM, Einevoll GT (2006) Currentsource density estimation based on inversion of electrostatic forward solution: effects of finite extent of neuronal activity and conductivity discontinuities. J Neurosci Methods 154:116-133. CrossRef Medline

Pinto L, Goard MJ, Estandian D, Xu M, Kwan AC, Lee SH, Harrison TC, Feng G, Dan Y (2013) Fast modulation of visual perception by basal forebrain cholinergic neurons. Nat Neurosci 16:1857-1863. CrossRef Medline

Polack PO, Friedman J, Golshani P (2013) Cellular mechanisms of brain state-dependent gain modulation in visual cortex. Nat Neurosci 16:13311339. CrossRef Medline

Poort J, Khan AG, Pachitariu M, Nemri A, Orsolic I, Krupic J, Bauza M, Sahani M, Keller GB, Mrsic-Flogel TD, Hofer SB (2015) Learning enhances sensory and multiple non-sensory representations in primary visual cortex. Neuron 86:1478-1490. CrossRef Medline

Reimer J, Froudarakis E, Cadwell CR, Yatsenko D, Denfield GH, Tolias AS (2014) Pupil fluctuations track fast switching of cortical states during quiet wakefulness. Neuron 84:355-362. CrossRef Medline

Reimer J, McGinley MJ, Liu Y, Rodenkirch C, Wang Q, McCormick DA, Tolias AS (2016) Pupil fluctuations track rapid changes in adrenergic and cholinergic activity in cortex. Nat Commun 7:13289. CrossRef Medline

Rossant C, Kadir SN, Goodman DF, Schulman J, Hunter ML, Saleem AB, Grosmark A, Belluscio M, Denfield GH, Ecker AS, Tolias AS, Solomon S, Buzsáki G, Carandini M, Harris KD (2016) Spike sorting for large, dense electrode arrays. Nat Neurosci 19:634-641. CrossRef Medline

Salazar RF, Dotson NM, Bressler SL, Gray CM (2012) Content-specific fronto-parietal synchronization during visual working memory. Science 338:1097-1100. CrossRef Medline

Schmiedt JT, Maier A, Fries P, Saunders RC, Leopold DA, Schmid MC (2014) Beta oscillation dynamics in extrastriate cortex after removal of primary visual cortex. J Neurosci 34:11857-11864. CrossRef Medline

Schroeder CE, Lakatos P (2009) Low-frequency neuronal oscillations as instruments of sensory selection. Trends Neurosci 32:9-18. CrossRef Medline
Seol GH, Ziburkus J, Huang S, Song L, Kim IT, Takamiya K, Huganir RL, Lee HK, Kirkwood A (2007) Neuromodulators control the polarity of spiketiming-dependent synaptic plasticity. Neuron 55:919-929. CrossRef Medline

Shobe JL, Claar LD, Parhami S, Bakhurin KI, Masmanidis SC (2015) Brain activity mapping at multiple scales with silicon microprobes containing 1,024 electrodes. J Neurophysiol 114:2043-2052. CrossRef Medline

Shuler MG, Bear MF (2006) Reward timing in the primary visual cortex. Science 311:1606-1609. CrossRef Medline

Siegle JH, López AC, Patel YA, Abramov K, Ohayon S, Voigts J (2017) Open Ephys: an open-source, plugin-based platform for multichannel electrophysiology. J Neural Eng 14:045003. CrossRef Medline

Stefanics G, Czigler I (2012) Automatic prediction error responses to hands with unexpected laterality: an electrophysiological study. Neuroimage 63: 253-261. CrossRef Medline

Stefanics G, Kimura M, Czigler I (2011) Visual mismatch negativity reveals automatic detection of sequential regularity violation. Front Hum Neurosci 5:46. CrossRef Medline

Stefanics G, Kremláček J, Czigler I (2014) Visual mismatch negativity: a predictive coding view. Front Hum Neurosci 8:666. CrossRef Medline

van Kerkoerle T, Self MW, Dagnino B, Gariel-Mathis MA, Poort J, van der Togt C, Roelfsema PR (2014) Alpha and gamma oscillations characterize feedback and feedforward processing in monkey visual cortex. Proc Natl Acad Sci U S A 111:14332-14341. CrossRef Medline

Vandecasteele M, Varga V, Berényi A, Papp E, Barthó P, Venance L, Freund TF, Buzsáki G (2014) Optogenetic activation of septal cholinergic neurons suppresses sharp wave ripples and enhances theta oscillations in the hippocampus. Proc Natl Acad Sci U S A 111:13535-13540. CrossRef Medline

Vinck M, Batista-Brito R, Knoblich U, Cardin JA (2015) Arousal and locomotion make distinct contributions to cortical activity patterns and visual encoding. Neuron 86:740-754. CrossRef Medline

Vinken K, Vogels R, Op de Beeck H (2017) Recent visual experience shapes visual processing in rats through stimulus-specific adaptation and response enhancement. Curr Biol 27:914-919. CrossRef Medline

Voloh B, Valiante TA, Everling S, Womelsdorf T (2015) Theta-gamma coordination between anterior cingulate and prefrontal cortex indexes correct attention shifts. Proc Natl Acad Sci U S A 112:8457-8462. CrossRef Medline

Wilson DA, Fletcher ML, Sullivan RM (2004) Acetylcholine and olfactory perceptual learning. Learn Mem 11:28-34. CrossRef Medline

Womelsdorf T, Fries P, Mitra PP, Desimone R (2006) Gamma-band synchronization in visual cortex predicts speed of change detection. Nature 439:733-736. CrossRef Medline

Zold CL, Hussain Shuler MG (2015) Theta oscillations in visual cortex emerge with experience to convey expected reward time and experienced reward rate. J Neurosci 35:9603-9614. CrossRef Medline 